\title{
Thermodynamics of voltage-gated ion channels
}

\author{
Xuejun C. Zhang ${ }^{1,2 \bowtie}$, Hanting Yang ${ }^{1}$, Zhenfeng Liu ${ }^{1,2}$, Fei Sun ${ }^{1,2}$ \\ 1 National Laboratory of Biomacromolecules, CAS Center for Excellence in Biomacromolecules, Institute of \\ Biophysics, Chinese Academy of Sciences, Beijing 100101, China \\ 2 College of Life Science, University of Chinese Academy of Sciences, Beijing 100049, China
}

Received: 25 April 2018 / Accepted: 15 July 2018 / Published online: 16 November 2018

\begin{abstract}
Ion channels are essential for cellular signaling. Voltage-gated ion channels (VGICs) are the largest and most extensively studied superfamily of ion channels. They possess modular structural features such as voltage-sensing domains that encircle and form mechanical connections with the pore-forming domains. Such features are intimately related to their function in sensing and responding to changes in the membrane potential. In the present work, we discuss the thermodynamic mechanisms of the VGIC superfamily, including the two-state gating mechanism, sliding-rocking mechanism of the voltage sensor, subunit cooperation, lipid-infiltration mechanism of inactivation, and the relationship with their structural features.
\end{abstract}

Keywords Ion channel, Gating mechanism, Membrane potential, Voltage sensor, Sliding-rocking model, Inactivation

\section{INTRODUCTION}

Information transmission in living cells is encoded through ion fluxes, resulting in complex spatiotemporal concentration patterns of ions. Being both a driving force and a result of these ion fluxes, the membrane potential is deeply intertwined with the dynamic distribution of ions across the membrane. Together, membrane potential and ion fluxes form an information network for regulation of cellular functions (Roux 1997). In this network, the transmembrane electrochemical potential is the major power source, and a variety of ions can be considered as the information carriers. Approximately $1 / 3-2 / 3$ of cellular energy originating from ATP hydrolysis is consumed to maintain the membrane potential (Howarth et al. 2012), in addition to other cellular resources used to establish and preserve the network infrastructure. In fact,

Electronic supplementary material The online version of this article (https://doi.org/10.1007/s41048-018-0074-y) contains supplementary material, which is available to authorized users.

$\triangle$ Correspondence: zhangc@ibp.ac.cn (X. C. Zhang), together with redox potential and ATP hydrolysis, membrane potential is one of the three major, interchangeable energy forms in all of living cells (Smith and Morowitz 2016). Without a properly maintained membrane potential, the cell would lose its essential information network. Similarly, ion channels, together with ion-sensing proteins as well as downstream effectors, are equally important, as they constitute basic functional nodes of such information network, perceiving, processing, and transducing signals encoded in ion fluxes (Jan and Jan 1989).

Ion channels are essential for transmembrane signaling and ionic homeostasis as well as regulating membrane polarization (Yellen 2002). They function in the plasma membrane, membranes of certain intracellular organelles, and other dynamic systems mediating cooperativities between organelles (Hosaka et al. 2017; Kintzer and Stroud 2017; Wei et al. 2016; Xu and Ren 2015; Yang et al. 2016). Ion channels are fundamentally different from another type of membrane proteins, namely transporters. While transporters are mainly responsible for transmembrane transport of metabolites and other substances such as nutrition uptake, ion channels are used for transducing ion-encoded 
information. In fact, the ions flowing through channels need to be replenished later by transporters or ion pumps. Such pumps are powered either directly by ATP hydrolysis or by transmembrane electrochemical potential of other substances (e.g., protons). Moreover, an ion channel differs from a transporter in its ability to transduce its substrate ions at a rate a few magnitudes larger than that of a transporter. Importantly, the substrate translocation pathway of a canonical ion channel opens to both sides of the membrane simultaneously. In contrast, a typical transporter opens to only one side at a given time through the alternating access mechanism (Jardetzky 1966). As the ion flux through a channel is driven by an electrochemical potential, whose maintenance renders fully functioning transporters an essential prerequisite, the signaling function of channels presumably appeared in the evolutionary course later than active transporters; nevertheless, this order remains to be a subject of debate (Pohorille 2005; Saier 2016). Interestingly, certain types of transporters can be converted into channels with limited structural modifications, as exemplified by the relationship between proton-pump bacteriorhodopsins and the light-evoked channel-rhodopsin from motile microalgae (Deisseroth and Hegemann 2017) and by that between canonical ABC exporters and the ATP-gated channel CFTR (Liu et al. 2017). Since the kinetic rates (e.g., activation rate) of a channel are high compared to that of transporters, conformational changes in the functional cycle of a channel are likely to be relatively small. In addition, persistent opening of a channel would result in depletion of cellular resources and thus be detrimental to cell viability. Therefore, channels are tightly regulated in terms of both substrate selectivity and permeability, and thus remain in their open state only transiently. A plethora of mechanisms for regulating channels has been discovered, including the gating mechanisms by voltage (Yellen 2002), ligand binding (Miller and Aricescu 2014; Wei et al. 2016), pH (Thompson et al. 2008), phosphorylation (Liu et al. 2017), mechanical force (Haswell et al. 2011; Zhang et al. 2016), photon (Deisseroth and Hegemann 2017), and temperature (Memon et al. 2017). A given ion channel can be regulated through multiple gating mechanisms, thus participating in complex signaling networks.

As fundamental functional units on the membrane of excitable cells, voltage-gated ion channels (VGICs) are crucial for electrical signaling in nerves and muscle cells among others. The VGIC superfamily includes members of the $\mathrm{K}^{+}-\left(\mathrm{K}_{\mathrm{V}}\right), \mathrm{Na}^{+}-\left(\mathrm{Na}_{\mathrm{V}}\right), \mathrm{Ca}^{2+}{ }_{-}, \mathrm{Cl}^{-}$, , and protonchannel families (Jan and Jan 1989; Purves et al. 2001; Tu et al. 2018), and has been extensively characterized through functional and structural approaches. Because of the vast volume of research literature on VGICs, we confine ourselves here to discuss the relationship between their common structural features and thermodynamic bases of their common mechanisms such as gating and voltage sensing. For more extensive discussions on the structure and function of ion channels as well as their physiological and clinical implications, readers are referred to excellent reviews published earlier, for examples Refs. (Bagneris et al. 2015; Catterall 2000; Jan and Jan 1989; Kintzer and Stroud 2017; Tombola et al. 2005a; Yellen 2002; Yu et al. 2005).

\section{OVERALL STRUCTURES OF CANONICAL VGICS}

For the sake of the following mechanistic discussion, we briefly summarize the common structural features of canonical, tetrameric VGICs (or simply referred here to as ion channels), using $\mathrm{K}^{+}$and $\mathrm{Na}^{+}$channels as examples. A typical tetrameric ion channel is composed of four, identical (or homologous), symmetrically assembled, transmembrane subunits (or repeats) (Fig. 1). Each subunit of the homo-tetrameric channel contains either two or six transmembrane (TM) helices. In a 2TM subunit, such as the KcsA channel from Streptomyces lividans, the two TM helices are termed outer and inner helices (Doyle et al. 1998), and in a 6TM subunit, such as the rat $\mathrm{K}_{\mathrm{V}} 1.2$ channel, the TM helices are termed S1S6 (Long et al. 2005). The S5 and S6 helices of the 6TM subunit are equivalent to the outer and inner helices of the 2TM subunit, respectively. In particular, the inner (S6) helices form a fourfold symmetrical, ion-conducting, central pore, and thus are called pore-lining helices. Therefore, the 6TM subunit contains, at its C-terminal region, a pore domain homologous to the 2TM subunit, with additional N-terminal S1-S4 helices forming a voltage-sensor (VS) domain. A subunit of the tetrameric 6TM channel either has its pore domain and VS domain in direct contact with each other or swaps its VS domain with neighboring subunits. In addition, two or four copies of the 6TM subunits may fuse into one peptide and become diversified between these repeats. The resulting pseudo-tetrameric channels contain only one or two such peptides (Kintzer and Stroud 2017; Yellen 2002). Certain channels employing more sophisticated regulatory mechanisms often contain additional domains, which may be significantly larger than the 6TM subunit itself (des Georges et al. 2016; Wei et al. 2016).

As it forms the central pore of the tetrameric ion channel, the 2TM subunit provides a suitable framework for understanding the common mechanisms of ion selectivity and gating in canonical tetrameric ion 

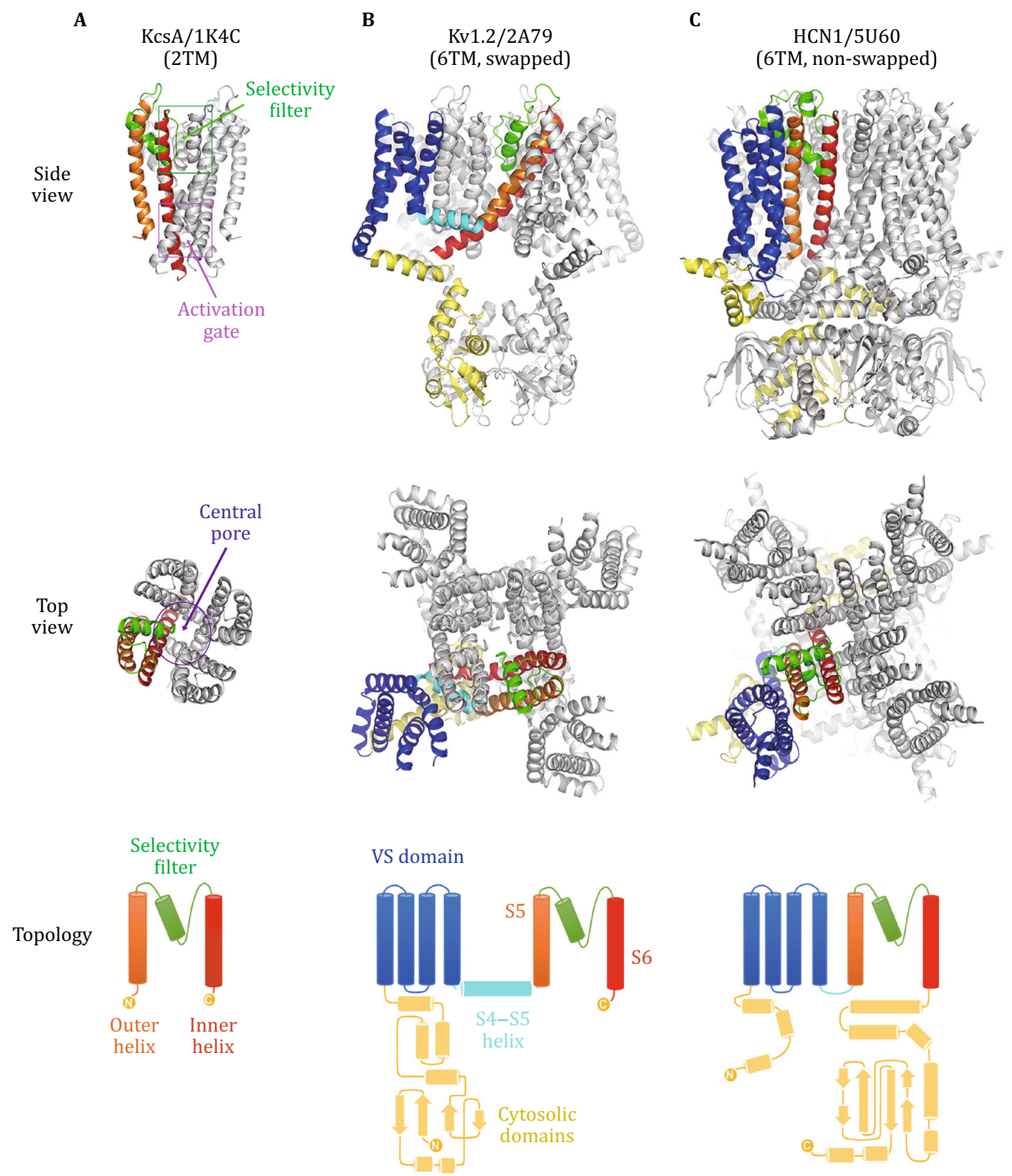

Fig. 1 Structures of tetrameric ion channels. A 2TM channel of KcsA/1K4C (Zhou et al. 2001). B Swapped 6TM channel of Kv1.2/2A79 (Long et al. 2005). C Non-swapped 6TM hyperpolarization-activated channel of HCN1/5U60 (Lee and MacKinnon 2017). From the top to bottom are the side view, top view, and topology diagrams. Structural elements from one subunit are colored as the following: VS domain, blue; S4-S5 helix, cyan; S5 (outer) helix, orange; selectivity-filter, green; S6 (inner) helix, red; and cytosolic domains, yellow. The other three subunits of the tetramer are shown in gray

channels. From an evolutionary point of view, this subunit very likely represents an ancient, primordial form of currently existing ion channels (Yu et al. 2005). In support of this argument, the 2TM subunit constitutes a number of essential structural features of the central pore. For example, both the $\mathrm{N}$ - and C-termini of this domain are located on the cytosolic side, defining the overall topology of the transmembrane helices. As one of the most noticeable structural features of all tetrameric ion channels, four inner (S6) helices form an "inverted teepee"-like core structure of the channel (viewed in the conventional orientation in which the cytosolic side is located at the bottom). This central core consists of three major layers: (1) The C-terminal 
segment of S6 helices bundle together, forming a righthandedly crossing, size-adjustable pore termed activation-gate, in the cytosolic $1 / 3$ region of the central pore. The inside wall of the activation-gate is typically lined with three rings of hydrophobic residues (Doyle et al. 1998), and this hydrophobicity is critical for the gating process (discussed below). (2) A short pore-helix (also called P-helix) and a pore-loop exist between S5 and $\mathrm{S} 6$ in the primary sequence of each subunit. At the level of the outer leaflet of the lipid bilayer, these P-helices and pore-loops fold inside the splayed part of the teepee-like structure. The pore-loop is stretched between the re-entrant P-helix and the $\mathrm{N}$-terminus of S6. Four pore-loops form a fourfold (pseudo-)symmetrical, polar selectivity-filter, in the extracellular $1 / 3$ of the central pore. In $\mathrm{K}^{+}$channels, only the backbone carbonyl groups of the pore-loop contribute to the binding of the substrates (McCoy and Nimigean 2012). For $\mathrm{Na}^{+}$and $\mathrm{Ca}^{2+}$ channels, an additional short helix, P2, is further inserted between the pore-loop and S6 in the primary sequence, and the selectivity-filter is formed by a mixture of side-chain polar groups and backbone carbonyl groups from the pore-loop. (3) An overall hydrophobic central cavity, which has a diameter larger than $10 \AA$, is located between the activation-gate and selectivity-filter in the tetrameric structure, constituting the middle $1 / 3$ region of the channel pore. This central cavity readily accommodates a cation together with its first hydration shell (roughly $8 \AA$ in diameter). In $\mathrm{K}^{+}$channels, this cavity is shown to be a strong ion binding site at the entrance of the selectivity-filter (Zhou et al. 2001). In addition, a negatively charged vestibule is often found around the entrance of a cation channel, functioning as a buffer zone to increase the local concentration of substrate cations (Payandeh et al. 2011). The structures of both the selectivity-filter and central cavity do not change significantly during channel gating (Bagneris et al. 2015; McCusker et al. 2012). Therefore, a consensus has been formed that most gating mechanisms are likely to operate through adjusting the physical and chemical properties of the hydrophobic activation-gate, which will be discussed in details below.

\section{ION MOVEMENT AND CONDUCTANCE}

Movement of the permeant substrate ions through a channel is driven by (and thus consumes) the transmembrane electrochemical potential of the substrates, including components of the membrane potential $\Delta \Psi_{\mathrm{M}}$ ( $\equiv \Psi_{\text {in }}-\Psi_{\text {out }}$ ) and/or concentration gradient of the substrate ions. The concentration gradient is commonly represented as the Nernst potential,
$V_{\mathrm{N}}\left(\equiv(R T / \mathrm{z} F) \cdot \ln \left([S]_{\text {in }} /[S]_{\text {out }}\right)\right.$, where $z$ is the charge valent of the substrate ion, and $R T / F \approx 25 \mathrm{mV}$ at $20^{\circ} \mathrm{C}$ ). Therefore, the overall effective transmembrane voltage, $\Delta \Psi^{\prime}\left(\equiv \Delta \Psi_{\mathrm{M}}+V_{\mathrm{N}}\right)$, represents the sole energy source driving substrate movement in nearly all ion channels.

Ion channels are arguably the best studied class of membrane proteins, largely because of the wellestablished biophysical techniques available for functional investigation. Among these techniques, patchclamp electrophysiology represents the "gold standard" for monitoring channel current, and provides quantitative information on the relationship between the transmembrane potential and ion movement (i.e., the electric current). In a patch-clamp study, the external voltage source, which replaces the membrane potential $\Delta \Psi_{\mathrm{M}}$, is controlled experimentally. After compensating $V_{\mathrm{N}}$, the ratio of the electric current $(I)$ to externally applied voltage $(V)$ at each point of the recorded $I-$ $V$ curve is, by definition, the macroscopic conductance of the channels (i.e., $G(V)$ as a function of voltage) (Fig. 1A). (Conductance is not to be confused with the Gibbs energy introduced below, also denoted as $G$ ). Once opened, each single channel is assumed to display a unitary single-channel conductance $\left(g_{\mathrm{sc}}\right)$, and its value was estimated to be in the range of $\sim 2-30 \mathrm{pS}$ for $\mathrm{K}^{+}$ channels (Gutman et al. 2005). At a $100-\mathrm{mV} \Delta \Psi_{\mathrm{M}}$, this range in conductance translates into a transient transporting rate of $\sim 10^{7}$ ions per second. In some rare cases, the $g_{\mathrm{sc}}$ appears to have multistep values, corresponding to distinct substrate selectivity and presumably being resulted from multi-conformations of the selectivity-filter (Zheng and Sigworth 1998). The macroscopic conductance is, thus, the product of the number of opened channels and $g_{\mathrm{sc}}$. The assumption of unitary $g_{\mathrm{sc}}$ for a given ion channel implies a microscopic two-state model, in which a single channel is either fully closed or fully opened rather than partially opened. Such a two-state model of channel gating has been supported by results from numerous voltage-clamp studies on a variety of single tetrameric ion channels (e.g., in Ren et al. 2001). Therefore, the normalized macroscopic conductance $\left(G / G_{\max }\right.$, where $G_{\max }$ represents the maximum conductance under a given experimental setup) equals to the channel-open probability (Zarrabi et al. 2010).

Hypothetically, an ideal linear $I-V$ relationship in a whole-cell electrophysiological experiment would indicate that the channels conduct substrate ions with a voltage-independent conductance. However, instead of displaying a perfect linear $I-V$ relationship, the macroscopic conductance of real channels always exhibits certain $\Delta \Psi_{\mathrm{M}}$-dependency for a number of reasons. First, no such linear relationship could be maintained if the 
magnitude of the applied voltage approached infinity. In fact, the ion current running through a single channel is limited by the diffusion rate of substrate ions from the surrounding into the buffer zone. Moreover, the structure as well as the function of a protein is strongly influenced by its environment. The most important environmental factor for an integral membrane protein, such as a channel, constitutes the membrane lipid bilayer. By adjusting the equilibrium position relative to the $\Delta \Psi_{\mathrm{M}}$-bearing membrane of a charge-carrying channel, the kinetic barrier of the channel and thus its $g_{\text {sc }}$ value may gradually change with changing of the membrane potential. In extreme cases, this type of regulation results in rectification of the $I-V$ curve. More generally, as long as it is not bound with the $\Delta \Psi_{\mathrm{M}}$, any charge-carrying membrane protein has the potential to function as a $\Delta \Psi_{\mathrm{M}}$-sensor. Nevertheless, the readout may not be always as evident as an ion channel in a voltage-clamp assay. While the theoretical possibility of such effects of $\Delta \Psi_{\mathrm{M}}$ on membrane proteins had been discussed several decades ago (Hill 1985), this type of interaction between membrane proteins and the $\Delta \Psi_{\mathrm{M}}$ is still not widely appreciated, especially in the field of studying the structures of membrane proteins. We argue that many long-range effects of mutations involving change of charges in membrane proteins might be explained as a consequence of their interaction with $\Delta \Psi_{\mathrm{M}}$. For example, a single-point mutation, $\mathrm{K} 1237 \mathrm{E}$, in the filter region of repeat-IV of $\mathrm{rNa}_{\mathrm{V}} 1.4$ resulted in a change of double charges, and this variant showed ultra-slow inactivation (Todt et al. 1999). This K1237E mutation probably has two effects, namely (1) "horizontal" repulsion between existing and introduced acidic residues inside the selectivity-filter, and (2) in the presence of $\Delta \Psi_{\mathrm{M}}$, a "vertical" electrostatic force (on the extra negative charges) which is likely to have a long-range effect on the conformation of the activationgate. In addition to the "gradual" change of $g_{\mathrm{sc}}$ with $\Delta \Psi_{\mathrm{M}}$, the activation-gate of a VGIC is able to abruptly switch between close and open states in response to the state transition of the VS domains. Taken together, the apparent conductance of a single ion channel is its "weakly" $\Delta \Psi_{\mathrm{M}}$-dependent single-channel conductance, $g_{\mathrm{sc}}$, modulated by the step-function of the VS activation.

Whereas $\Delta \Psi_{\mathrm{M}}$ directly exerts its power through interaction with the electric charges of the substrate ions, the other component of $\Delta \Psi^{\prime}, V_{\mathrm{N}}$, originates from the chemical potential (i.e., concentration gradient) of the substrate ions and appears to act indirectly. Mathematically, a force is defined as the decrease in energy across a specific distance. It is in this sense that the thermodynamic force of $V_{\mathrm{N}}$ becomes comparable with the deterministic force of $\Delta \Psi_{\mathrm{M}}$. Let us first look into the deterministic force of $\Delta \Psi_{\mathrm{M}}$. In the steady open state of the channel, the electrostatic energy of the ions is utilized to overcome the friction inside the selectivity-filter, thus dissipating as heat. Since the single-channel conductance remains constant, the ion movement $(I)$ is linearly proportional to the electrostatic force applied to the ions $(V)$, following the macroscopic Ohm law. Thus, the friction/damping coefficient (i.e., the ratio of the applied force to the speed of ion movement (Qian and Kou 2014)) remains constant at the microscopic level. Secondly, we look into the statistical force of $V_{\mathrm{N}}$. An ion that binds to the entrance of the selectivity-filter does not directly sense the concentration of the same type of ions at the other end of the filter and beyond. Rather, this ion senses the gradient through electrostatic repelling between ions. This electrostatic force is propagated along the selectivity-filter in a manner similar to Newton's cradle. Interestingly, substrates of almost all known selective channels are electrically charged. For simplicity, we consider only the electric potential between the two ends of the selectivity-filter of an ideal channel. In such an ideal channel, the two terminal binding sites are specific to the substrate ions, with negligible affinity to other ions; otherwise complicated competition with (and/or inhibition by) other substances must be included for kinetic consideration. In addition, the substrate specificity is determined not only by affinity at the two ends but also by the property of the selectivity-filter itself. For example, the selectivity-filter of a $\mathrm{K}^{+}$channel possesses an energetic trap for the substrate $\mathrm{K}^{+}$ions (as well as inhibitory ions), and the same selectivity-filter behaves as an energy barrier for other types of ions (Zhou et al. 2001). These detailed features of the energy landscape within the selectivity-filter are relevant only to the kinetics, but can be omitted from our thermodynamic consideration. Taken together, binding of an ion to one terminal site favors movement of ions inside the selectivity-filter towards the opposite terminal site. In the absence of $\Delta \Psi_{\mathrm{M}}$, the final direction of the movement inside the filter would be determined by the transmembrane gradient of the substrate concentration. This is analogous to the movement of a two-way door, in which the side of stronger pressure wins. In fact, the chemical potential of the substrate, $\Delta \mu(\mathrm{S}) \quad\left(\equiv R T \cdot \ln \left([\mathrm{S}]_{\mathrm{R}} /[\mathrm{S}]_{\mathrm{L}}\right)\right.$, where the subscripts $\mathrm{R}$ and $\mathrm{L}$ stand for releasing and loading, respectively, drives the substrate movement, regardless of the charge(s) carried by the substrate. In this scenario, the electrostatic forces between the charges of the substrate ions are only a medium for transmitting the information about the chemical potential, but are not the bona fide driving force. In the presence of $\Delta \Psi_{\mathrm{M}}$, the $\Delta \mu$-driven ion movement is either accelerated or slowed 
down (even reversed) by $\Delta \Psi_{\mathrm{M}}$, depending on the direction and strength of $\Delta \Psi_{\mathrm{M}}$ relative to the concentration gradient. Moreover, the average concentration of net charges to maintain $\Delta \Psi_{\mathrm{M}}$ is low compared to the local concentration associated with a tightly bound ion to a membrane protein. Assuming a value of $100-\mathrm{mV}$ for $\Delta \Psi_{\mathrm{M}}$ and a 30- $\AA$ thickness $(d)$ of the membrane, the net charge density $\left(Q / A=\Delta \Psi_{\mathrm{M}} C / A=\varepsilon_{0} \Delta \Psi_{\mathrm{M}} / d\right.$, where $A$ and $C$ stand for surface area and electric capacity, respectively) on each side of the membrane is estimated as being only $\sim 0.02 \mathrm{e}_{0}$ per $1000 A^{2}$ surface-equivalent to the cross section of a typical membrane protein. Therefore, binding of a single ion at one end of the selectivity-filter will strongly affect the electrical field within the selectivity-filter. In a real channel, however, such an effect is attenuated by differential binding of ions between the two opposite terminal sites. In this sense, $\Delta \mu(\mathrm{S})$ is equivalent to an imaginary transmembrane electrostatic voltage, $V_{\mathrm{N}}$. This Nernst voltage is only applicable to a conduction pathway that connects the two spaces defining the $\Delta \mu(\mathrm{S})$.

\section{DRY/WET TWO-STATE MODEL OF THE GATING PROCESS}

Thermodynamics of the gating process of the hydrophobic activation-gate has been described using a continuous-medium model (Anishkin et al. 2010; Anishkin and Sukharev 2004). In this simplified yet elegant two-state model, the activation-gate is represented by a cylindrical pore which has a hydrophobic inner surface. In the close state of the gate, the size (i.e., radius) of the cylinder is small, and its pore is narrow and "dry"; in contrast, in the open state, the size of the cylinder increases, and its pore turns "wet." More specifically, in the dry state, a vapor seal exists inside the cylinder and expels water and other polar solvent molecules as well as ions, and in the wet state, the hydrophobic cylinder becomes filled with aqueous solvent. Two conflicting energetic factors determine the state of the activation-gate cylinder (Fig. 2). On the one hand, the dry state maintains two aqueous-gas interfaces at the two ends of the vapor seal, and thus the dry state is associated with an energy term proportional to the area (or the radius square) of the cross section of the activation-gate. On the other hand, filling the hydrophobic activation-gate with aqueous solvent also consumes energy. This second energy term is proportional to the area of the wetted inner surface of the cylinder, and thus increases linearly with the radius of the pore cylinder. Therefore, whether the activation-gate is dry or wet depends on the radius of the activation-gate. Once the radius of the activation-gate becomes larger than the so-called "critical radius" of wetting ( $r_{\mathrm{c}}$, approx. $5 \AA$ (Anishkin et al. 2010)), the wet state of the activation-gate becomes less energetically costing than the dry state, the vapor seal breaks, and the cylinder pore is filled with polar molecules and ions. The open probability $P_{\mathrm{o}}(r)$ of the activation-gate as a function of the gate radius is determined by the differential Gibbs energy, $\Delta G_{\mathrm{dw}}$ ( $\equiv G_{\text {wet }}-G_{\mathrm{dry}}$ ), according to the Boltzmann distribution:

$P_{\mathrm{o}}(r)=\left[1+\exp \left(\Delta G_{\mathrm{dw}}(r) / R T\right)\right]^{-1}$.

At the critical radius, $\Delta G_{\mathrm{dw}}\left(r_{\mathrm{c}}\right)$ equals to zero, and the two states are evenly distributed. The $P_{\mathrm{o}}(r)$ curve is likely to be steep around the critical radius, meaning that a small structural change may have a large effect on the open probability of the activation-gate. As discussed below, the structural changes associated with $\Delta G_{\mathrm{dw}}$ are usually driven by other energy sources (e.g., the differential conformational energy of the activation-gate, $\Delta G_{\text {gating}}$ ). The concept that a small structural adjustment in the activation-gate region is able to trigger an instantaneous state transition of the activation-gate is consistent with rapid activation of ion channels (Bagneris et al. 2015). This two-state mechanism has been convincingly applied to mechanosensitive channels to explain their gating mechanism (Anishkin et al. 2010).

During gate opening, the four pore-lining S6 helices either physically move away from each other (thus increasing the "radius" of the activation-gate) or chemically increase the hydrophilicity of the activation-gate (thus reducing both the energy cost of wetting and $r_{\mathrm{c}}$ ). In any case, a necessary condition for the activation-gate to supply the selectivity-filter with a continuous ion flow is to have the cross section of the gate larger than the size of hydrated ions. In experimentally determined channel structures (e.g., in $\mathrm{Na}_{\mathrm{V}} \mathrm{Ms} /(\mathrm{PDB}$ IDs: 3Z)Z and 4F4L) (Bagneris et al. 2013; McCusker et al. 2012) both radially outward bending and rotation of S6 helices are observed, resulting in dilation of the activation-gate and increase of the hydrophilicity of the gate pore. These conformational changes are based on a pivot at the middle point of S6 (called gating-hinge, e.g., at the residue $\mathrm{T} 206$ in $\mathrm{Na}_{V} \mathrm{Ms}$ ), and were considered as structural evidence to support the original "hinge motion hypothesis" on channel gating (Zarrabi et al. 2010). However, this hypothesis alone may lead to a model of continuous gating. In contrast, combining it with the two-state model better accounts for the common observation of one-or-none conductance of a single channel.

How is the state change of the activation-gate translated into instantaneous channel opening? In the open 
A

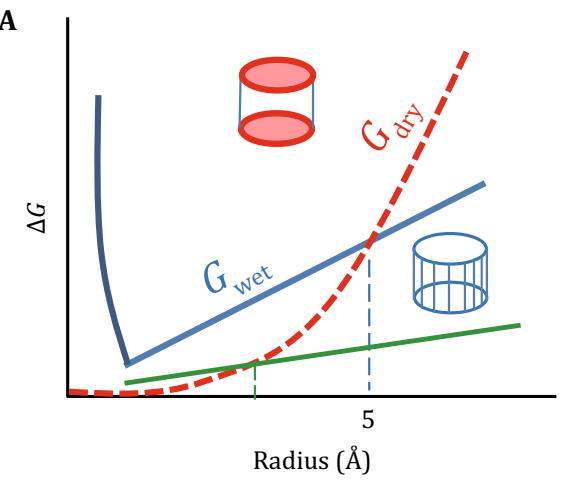

C

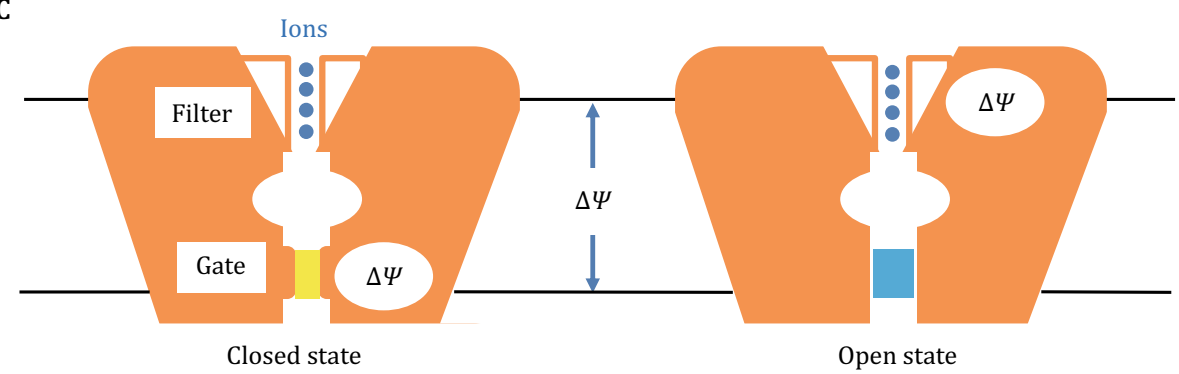

B

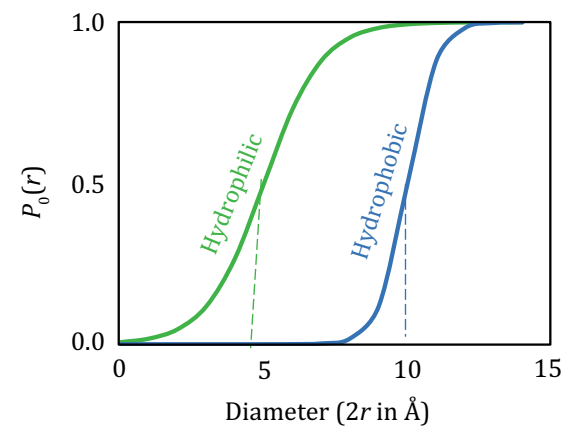

Fig. 2 Dry/wet two-state model of the activation-gate. A Gibbs free energies of the dry (red, labeled as $\left.G_{\text {dry }}\right)$ and wet (blue, $\left.G_{\text {wet }}\right)$ states as functions of the radius of the activation-gate. Their crossing point corresponds to the critical radius $\left(r_{\mathrm{c}}\right)$. Increasing the hydrophobicity of the gate decreases the slope of the $G_{\text {wet }}$ curve (green), resulting in a reduced critical radius. B Open probability of the gate, $P_{\mathrm{o}}(r)\left(\equiv\left[1+\exp \left(\left(G_{\text {wet }}-G_{\mathrm{dry}}\right) / R T\right)\right]^{-1}\right)$. Increasing the hydrophilicity of the gate shifts the $P_{\mathrm{o}}$ curve to a smaller $r_{\mathrm{c}}$. C Schematic diagram of the gating process. In the dry state (with a yellow vapor seal), $\Delta \Psi_{\mathrm{M}}$ is loaded on the activation-gate; in contrast, in the wet state (with cyan water filling), $\Delta \Psi_{\mathrm{M}}$ is loaded on the selectivity-filter

state of the channel, the movement of the substrate ions inside the channel is driven by $\Delta \Psi^{\prime}$. The central pore can be considered as an electric circuit of two resistors of the activation-gate and selectivity-filter. The voltagedrop on each part of the circuit is proportional to its resistance (i.e., reciprocal of the conductance) relative to the overall resistance of the series-connected circuit. In the dry state, the activation-gate has a very large resistance, practically approaching to infinity. Thus, the voltage-drop applied to the selectivity-filter is zero, because the activation-gate draws all of $\Delta \Psi_{\mathrm{M}}$. In this case, an induced electric dipole is formed by both cations trapped inside the selectivity-filter and anions attracted locally on the positive side of $\Delta \Psi_{\mathrm{M}}$. While this induced dipole cancels the electrical field of $\Delta \Psi_{\mathrm{M}}$ inside the selectivity-filter, it also strengthens the field across the activation-gate. Therefore, the electric current passing the selectivity-filter is zero, and consequently the channel is closed. Importantly, this closure functions only in the sense of electric conduction, because the physical size of the activation-gate may remain sufficiently large to hold a hydrated ion (though unable to do so because of the vapor seal). In contrast, in the wet state, the activation-gate is filled with solvent exhibiting a high dielectric constant. Water molecules inside the activation-gate become polarized by the external electrical field of $\Delta \Psi_{\mathrm{M}}$, and their induced dipoles (partially) cancel the external electrical field. Consequently, the total electrical field inside the activation-gate approaches to zero, and nearly the entire $\Delta \Psi_{\mathrm{M}}$ is focused on the selectivity-filter, driving the ion movement. Because of this electric nature of the gating process, a small structural change around the critical radius of $P_{\mathrm{o}}(r)$ instantaneously reshapes the electrostatic field of $\Delta \Psi_{\mathrm{M}}$, and thus generates a quick response of the channel current to the activation cues that regulate the pore size.

\section{VOLTAGE SENSOR}

\section{Sliding versus rocking models}

In the voltage-gated, tetrameric 6TM, ion channels, the voltage sensor (VS) is comprised of transmembrane helices S1-S4. $\Delta \Psi_{\mathrm{M}}$ determines the conformation of the VS domain, and thus the change of $\Delta \Psi_{\mathrm{M}}$ (i.e., $\Delta \Delta \Psi_{\mathrm{M}}$ ) is responsible for the conformational change. In response to $\Delta \Delta \Psi_{\mathrm{M}}$, the VS domain regulates the opening of the 
activation-gate. Homologous functional VS domains are also found in non-channel membrane proteins (Sakata et al. 2016), suggesting that these proteins utilize a common voltage-sensing mechanism conferred by the VS domains. For most known VGICs, the open probability $\left(P_{\mathrm{o}}(V)\right)$ of the activation-gate increases as the $\Delta \Psi_{\mathrm{M}}$ is depolarized from its resting value. Thus, this type of channels is specifically called depolarization-activated channels. In contrast, for hyperpolarization-activation cyclic nucleotide-gated (HCN) channels, the activationgate is opened when the $\Delta \Psi_{\mathrm{M}}$ becomes more polarized (Novella Romanelli et al. 2016). This latter type of channels is responsible for restricting the resting $\Delta \Psi_{\mathrm{M}}$ and for pace-making in cardiac cells (Jackson et al. 2007). Although the VS domains from the two types of channels show distinct effects in response to membrane polarization, they are homologous in their primary sequences and share similar overall 3D structures (Jackson et al. 2007; Lee and MacKinnon 2017). More specifically, VS domains from both types of voltagegated channels contain a conserved, highly positively charged S4 helix, part of which assumes a $310^{\text {-helix }}$ conformation (Long et al. 2005). Because of this $3_{10}$ conformation, 4-6 basic residues residing on S4 (referred to as R0-R5) are aligned on the same side of the helix surface. For example, in $\mathrm{Na}_{\mathrm{v}}$ channels, four conserved Arg residues (R1-R4) are observed in S4 (Bagneris et al. 2015). These basic residues are believed to contribute to the gating-charges of the VS domain (Armstrong and Bezanilla 1973; Bagneris et al. 2015; Islas and Sigworth 2001; Mannuzzu et al. 1996; Stuhmer et al. 1989).

Unlike a selectivity-filter, the VS domain does not sense the Nernst potential. In fact, in the time scale of conformational changes of a VS domain, the electric field of ions responsible for the Nernst potential is effectively screened out by the surrounding solution including counter ions; however, the same argument would not be true for the microscopic environment of a selectivefilter. Thus, a leak-free VS domain changes its conformation in response only to $\Delta \Delta \Psi_{\mathrm{M}}$, but not to $V_{\mathrm{N}}$ (Benedek and Villars 2000). Upon depolarization of the membrane potential, the VS domain undergoes a rather large conformational transition, translocating positive gating-charges from the cytosolic side to the extracellular side of the $\Delta \Psi_{\mathrm{M}}$. The precise mechanism of the charge translocation remains under debate. A currently favored hypothesis is that the positively charged S4 undergoes a large outward sliding movement relative to the membrane (Catterall 2010). This sliding mechanism is consistent with results from disulfide-bond formation experiments, which trapped a few putative intermediate states during activation of the VS domain (DeCaen et al.
2009), and with the high degree of conservation of the R1-R4 residues aligning on the S4 surface. Nevertheless, direct structure evidence for such sliding hypothesis has not been reported thus far. An alternative chargetranslocating mechanism is the rocking-like two-state model similar to that of many transporters. In such a rocking model, the conformation of the VS domain changes between an outward-facing conformation $\left(\mathrm{C}_{\text {out }}\right)$ and an inward-facing conformation $\left(\mathrm{C}_{\mathrm{in}}\right.$, where the in/ out orientation is referred to channels on the plasma membrane) (Chanda et al. 2005; Kintzer and Stroud 2017). This rocking mechanism is supported by a fluorescence experiment showing that the S4 helix does not move significantly relative to the membrane lipid bilayer (Chanda et al. 2005). In addition, certain histidine substitutions of arginine residues in S4 are shown to convert the VS domain into a proton transporter, suggesting that a voltage sensor and a transporter mechanistically function in a similar manner (Starace and Bezanilla 2001). It should be noted that both the sliding model and the rocking model appear to agree with results of the studies conducted to investigate chemical reactivity of cysteine mutations (Yang and Horn 1995). Other studies using voltage-clamp fluorometry (Cha and Bezanilla 1997; Mannuzzu et al. 1996) indicated that upon the change of membrane potential, a large conformational change within VS results in the translocation of gating-charges (Catterall 2010). A major question concerning the difference between the two models is whether the conformational change is via sliding of $\mathrm{S} 4$ relative to the membrane (Catterall 2010; Ruta et al. 2005) or a rocking-like motion (Yellen 2002).

Based on currently available data, we propose an alternative putative mechanism, called sliding-rocking two-state model (Fig. 3): a VS domain uses a hybrid mechanism of the above two models. In this combined model, the sliding of $\mathrm{S} 4$ is relative to a polar slot formed by S1-S3, but not to the membrane per se, being accompanied by a rocking motion between the two parts of VS. In the research field of transporters, a similar mechanism is called an "elevator car" mechanism (McCoy et al. 2016). During the sliding-rocking movement of the VS domain, two crevices are created alternatingly on the two sides of the membrane, resulting in drastically reshaping of the electrical field of $\Delta \Psi_{\mathrm{M}}$ inside the VS domain and at the same time translocating the gating-charges relative to the electrical field.

\section{Structural changes between the two states}

Currently, the only VS domain for which the structures of both inactive and active states have been reported 
A

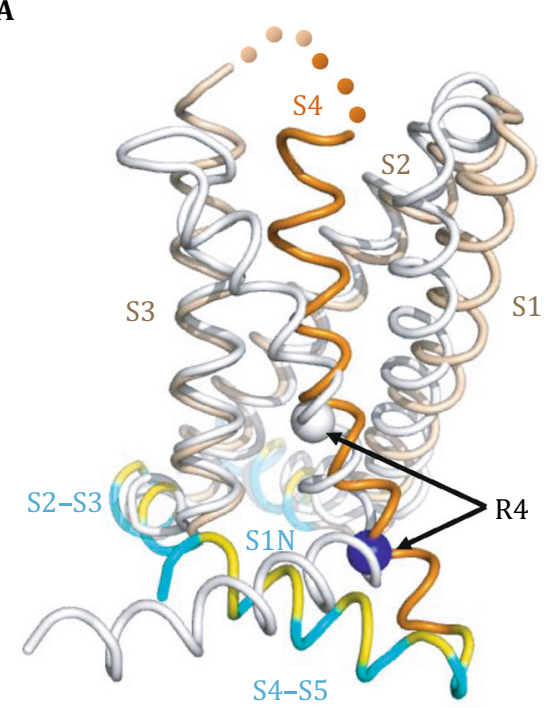

B

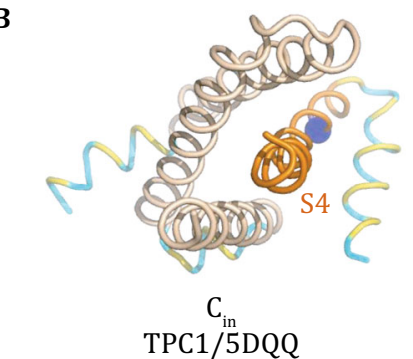

C

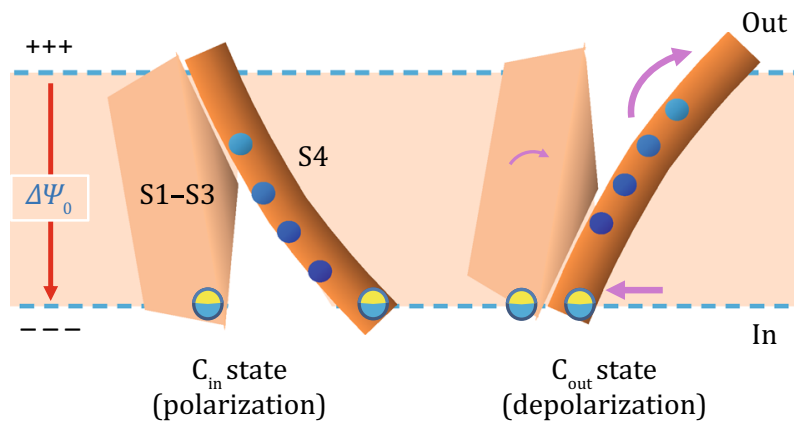

Fig. 3 Sliding-rocking two-state model of the voltage-sensor domain. A Superposition of VS domains from TPC1/5DQQ ( $\mathrm{C}_{\text {in }}$ state) and $\mathrm{Na} \mathrm{V}_{\mathrm{V}} \mathrm{Ab} / 3 \mathrm{RVY}\left(\mathrm{C}_{\text {out }}\right)$. The $\mathrm{C}_{\text {in }}$ structure is colored in wheat color (S1, S2, and S3), orange (S4), and cyan-yellow mixed (amphipathic helices), and the $\mathrm{C}_{\text {out }}$ structure is colored in gray for clarity. Positions of one of the four conserved basic residues, R4, in the two structures are marked with blue and gray spheres, indicating a two-turn sliding movement of S4 relative to the remaining VS domain. S2 and S3 as well as the amphipathic linker helix (S2-S3) are superimposed well. B Extracellular view of the VS domains shown in panel (A). C Schematic diagram of the sliding-rocking model. In the polarization state, the VS domain has an inward-facing conformation $\left(\mathrm{C}_{\mathrm{in}}\right)$. Upon depolarization, the outward-facing conformation $\left(\mathrm{C}_{\text {out }}\right)$ becomes energetically favored. The voltage change is sensed by positively charged residues in S4, R1-R4 marked as spheres from light to dark blue. Movements of structural elements are indicated by magenta arrows. During the conformational change, the cytosolic ends of S1-S4 are anchored to the cytosolic surface of the membrane by amphipathic helices (depicted as yellow-cyan circles)

was isolated from a voltage-sensing phosphatase from Ciona intestinalis (Ci-VSD) (Li et al. 2014). As the registration of amino acid sequences (especially that of S4) into weak electron densities is ambiguous, the presumed "inactive" $C_{i n}$ structural model of Ci-VSD remains uncertain. Moreover, whereas the experimental $\mathrm{C}_{\text {out }}$ structures of VS domains have been repeatedly reported [e.g., Kv1.2/2A79 (Long et al. 2005) and $\mathrm{Na}_{\mathrm{V}}$ $\mathrm{Ab} / 3 \mathrm{RVY}$ (Payandeh et al. 2011)], structural information of the $\mathrm{C}_{\mathrm{in}}$ state is limited to that of TPC1/(5DQQ and 5E1 J) from Arabidopsis thaliana (Guo et al. 2016; Kintzer and Stroud 2016). The dominance of $\mathrm{C}_{\text {out }}$ states in reported structural studies may be partially explained by the lack of $\Delta \Psi_{\mathrm{M}}$ under experimental conditions for the structural data reported.

In both $\mathrm{C}_{\text {in }}$ and $\mathrm{C}_{\text {out }}$ structures of VS domains (Fig. 3A), the S4 helix is slightly curved, with its positively charged, convex surface facing the remaining part of the VS domain. The R1-R4 residues are located in the 5th-2nd turns (counted from the C-terminal end of S4), respectively. In the in vivo membrane environment, the C-terminal end of S4 is likely anchored to the cytosolic surface of the membrane by the following amphipathic
S4-S5 helix. Moreover, in both $\mathrm{C}_{\text {in }}$ and $\mathrm{C}_{\text {out }}$ structures, S1, S2, and S3 form a transmembrane hydrophilic slot, with an "N"-shaped topology for the peptide chain. This slot provides the positively charged S4 surface with a low energy-barrier sliding track for translocation of the gating-charges. The overall hydrophilic property of their interface is further supported by the observation that a single-point mutation at R1 created a proton-wire across the TM region of the VS domain (Starace and Bezanilla 2001). In the middle of the $\mathrm{N}$-shaped slot is the so-called gating-charge-transfer center (Tao et al. 2010), which contains both a conserved negatively charged residue partially neutralizing positive charges from S4 and a conserved hydrophobic residue acting as a seal to prevent charge leakage.

Structural comparison of the two states provides further support to the sliding-rocking model. In the $\mathrm{C}_{\text {out }}$ structure of $\mathrm{Na}_{\mathrm{V}} \mathrm{Ab} / 3 \mathrm{RVY}$, the S1-S2 loop (i.e., the loop connecting S1 and S2) separates from the extracellular end of S4, while the four helices S1-S4 are bundled together within the intracellular region (Fig. 3). The separation between S1-S2 and S4 creates an aqueous crevice $(\sim 10-\AA \AA$ or deeper $)$ penetrating from the 
extracellular space into the TM region. Three of the positively charged residues in $\mathrm{S} 4\left(\mathrm{R} 99^{(\mathrm{R} 1)}, \mathrm{R} 102^{(\mathrm{R} 2)}\right.$, and $\mathrm{R} 105^{(\mathrm{R} 3)}$ of $\mathrm{Na}_{\mathrm{V}} \mathrm{Ab}$ ) face the interior of this crevice, and are thus all located on the positive side of $\Delta \Psi_{\mathrm{M}}$. In comparison, in the $\mathrm{C}_{\text {in }}$ structure of TPC1/5DQQ the S1S4 helices of the functional VS-II domain become bundled together in the extracellular region; the C-terminal half of S4 separates from the S1-S3 helix bundle in the intracellular region. This structural rearrangement eliminates the extracellular crevice, but instead creates an aqueous crevice (deeper than $10-\AA ̊)$ penetrating from the cytosolic side into the TM region. Three of the positively charged residues in S4 $\left(\mathrm{R} 537^{(\mathrm{R} 2)}, \mathrm{R} 540^{(\mathrm{R} 3)}\right.$, and $\mathrm{R} 543^{(\mathrm{R} 4)}$ of TPC1) face the interior of the intracellular crevice, and are thus all located on the negative side of $\Delta \Psi_{\mathrm{M}}$. The rearrangement of S4 relative to S1-S3 during the $\mathrm{C}_{\text {in }}$-to- $\mathrm{C}_{\text {out }}$ (I-O) transition results in a twoturn sliding towards the extracellular direction (Fig. 3A) (She et al. 2018), in agreement with the observation from the disulfide-bonding experiment (DeCaen et al. 2009). This sliding movement of $S 4$ requires local conformational change in the S3-S4 linker. In fact, this linker region is invisible in several VS crystal structures, indicating high flexibility, and varies in both amino acid sequence and length between species. By probing with a variety of neurotoxins, the extracellular S3-S4 loops in several VGICs are shown to assume different conformations between the resting and activated states (Catterall 2010). With increasingly larger truncation in this loop, mutants of the Shaker $K_{\mathrm{V}}$ channel showed slower activation rates and more right-shifts of the activation curve, indicating that the channel becomes harder to open (Gonzalez et al. 2000). In comparison, the linker between the C-terminal end of S4 helix and the S4-S5 amphipathic helix is always well defined in the crystal structures, although the angle between the two helices changes from $\sim 35^{\circ}$ in $\mathrm{C}_{\text {in }}$ to $\sim 80^{\circ}$ in $\mathrm{C}_{\text {out }}$ (Fig. 3A). It is important to re-emphasize that, during conformational transitions between $\mathrm{C}_{\text {in }}$ and $\mathrm{C}_{\text {out }}$, the $\mathrm{C}$-terminal end of S4 likely remains anchored to the intracellular surface of the membrane by the S4-S5 amphipathic helix. Similarly, in a number of ion channels, the N-terminal end of S1 is also found to be linked to a short amphipathic helix, S1 N, and S2 and S3 are connected by a short amphipathic helix S2-S3. Together, these amphipathic anchors on the intracellular surface impose strong restraints on possible conformational changes of the VS domain. For the experimentally observed conformational change to occur in a membrane environment while keeping the anchoring in effect, the two parts of VS, i.e., S4 and the S1-S3 bundle, are mostly likely to rock relatively to each other as well as to the membrane (Fig. 3C). Consequently, the structural difference between $\mathrm{C}_{\text {in }}$ and $\mathrm{C}_{\text {out }}$ results in translocation of the gating-charges relative to the focused electrical field of $\Delta \Psi_{\mathrm{M}}$, supporting the sliding-rocking mechanism of the VS domain.

As a side note, the $A$. thaliana channel TPC1 is interesting in several aspects, besides providing structural information on the $\mathrm{C}_{\text {in }}$ state. TPC1 is composed of a homodimer, and each of the two subunits contains two VS-like domains (VS-I and VS-II). Of the four putative VS domains, only two diagonally arranged VS-II domains are functional in voltage sensing. Activation of the channel requires both the voltage sensing by the two VS-II domains and $\mathrm{Ca}^{2+}$ binding to cytosolic domains which are attached to the pseudo-VS-I domains (Guo et al. 2016). Furthermore, binding of $\mathrm{Ca}^{2+}$ on the extracellular/vesicle-lumen side of the VS-II domains in the $C_{\text {in }}$ state inhibits the channel opening (Guo et al. 2016). This inhibitory effect is probably a consequence of locking together the S1-S2 and S3-S4 loops, and also a result of exerting an extra inward electrostatic force on the VS-II domain, both stabilizing the $\mathrm{C}_{\text {in }}$ state.

\section{Gating-charges}

The basic residues residing along the $\mathrm{S} 4$ helix are proved to be critical for voltage sensing (Catterall 2000, 2010). The inward electrostatic forces that are exerted on these basic residues increase with polarization of the negative-inside $\Delta \Psi_{\mathrm{M}}$. Similarly, other charged residues residing on S2 and S3 (mostly being conserved acidic residues) also sense $\Delta \Delta \Psi_{\mathrm{M}}$. These changes of electrostatic forces are responsible for the relative movements between S4 and the S1-S3 bundle, switching the VS domain between $\mathrm{C}_{\text {out }}$ and $\mathrm{C}_{\mathrm{in}}$. Whereas the conserved positively charged residues concentrated in S4, namely R1-R4, are likely to be the main contributors to the overall gating-charges, all charged residues contribute to the gating-charges with varied weights (Online Appendix) (Islas and Sigworth 2001). More specifically, whether a charged residue makes a significant contribution to the overall gating-charges ultimately depends on its positional shift relative to the electrical field of $\Delta \Psi_{\mathrm{M}}$ during the conformational transition of the VS domain.

Another important force exerted on membrane proteins is the hydrophobic mismatch (Phillips et al. 2009), which originates from a positional mismatch between the hydrophobic surface of the membrane protein and the surrounding lipid bilayer. Like electrostatic forces, the forces generated by hydrophobic mismatches also change in magnitude and even direction in response to $\Delta \Delta \Psi_{\mathrm{M}}$, keeping the protein to reside inside the membrane bilayer. The relationship between the electrostatic 
force and hydrophobic mismatch can be analogous to the interaction between a floating boat and its surrounding water. Just as the gravitational force pulling the boat downwards is balanced by the buoyancy force that keeps the boat afloat, the $\mathrm{C}_{\text {in }}$ state of a VS domain is maintained by the balance between forces of hydrophobic mismatch and the inward electrostatic forces exerted by $\Delta \Psi_{\mathrm{M}}$ on each and every positively charged residue (as well as the outward electrostatic forces on negatively charged residues). Upon $\Delta \Psi_{\mathrm{M}^{-}}$ depolarization, the inward (outward) electrostatic forces exerted on basic (acidic) residues are reduced; the mechanical balance of the $\mathrm{C}_{\text {in }}$ state is broken; distinct mechanical torques are generated on different helices of the VS domain, promoting their relative movements. As a result, the overall conformation of the VS domain changes, and in the process a new state, $\mathrm{C}_{\text {out }}$, equilibrating between electrostatic and hydrophobic forces is established. Importantly, during the state transition, the tilting angle of S4 relative to the membrane normal is likely to change, a process that is accompanied by distinct patterns of charge exposure. A similar $\Delta \Psi_{\mathrm{M}}$-driven conformational change has been proposed for proton motive force (PMF)-driven transporters, referred to as membrane potential-driving hypothesis (Zhang et al. 2015,2017 a), which is an extension and realization in transmembrane transport of the chemiosmotic theory proposed by Peter Mitchell. However, these two types of membrane proteins exhibit one major difference: in PMF-driven transporters, it is the change of electric charge (associated with protonation and deprotonation) that drives the transport cycle. In contrast, in the VS domain, it is the change of membrane potential that drives the conformational change during voltage sensing.

In addition to the alternating patterns of gatingcharge exposure, each of the $\mathrm{C}_{\text {in }}$ and $\mathrm{C}_{\text {out }}$ states seems to be stabilized by a distinct group of interaction within the VS domain, including salt-bridge bonds. For instance, in the $\mathrm{C}_{\text {out }}$ structure of $\mathrm{Na}_{\mathrm{V}} \mathrm{Ab} / 3 \mathrm{RVY}$, residues $\mathrm{E} 59^{\mathrm{S} 2}, \mathrm{R} 63^{\mathrm{S} 2}, \mathrm{D} 80^{\mathrm{S} 3}$, and $\mathrm{R} 108^{\mathrm{S} 4(\mathrm{R} 4)}$ form a charge cluster in the cytosolic half of the VS domain. It can be envisioned that, in the $\mathrm{C}_{\text {in }}$ state, these interactions are lost; instead, new interactions are formed in the extracellular half of the VS domain, including a salt-bridge bond between $\mathrm{E} 32^{\mathrm{S} 1}$ and $\mathrm{R} 99^{\mathrm{S4}(\mathrm{R} 1)}$. Similarly, in the $\mathrm{C}_{\text {in }}$ structure of $\mathrm{TPC} 1 / 5 \mathrm{DQQ} \quad \mathrm{E} 511^{\mathrm{S} 3}-\mathrm{R} 531^{\mathrm{S} 4(\mathrm{R} 0)}$ and $\mathrm{D} 500^{\mathrm{S3}}-\mathrm{R} 540^{\mathrm{S4}(\mathrm{R} 3)}$ pairs are observed to form two saltbridge bonds. These distinct clusters of charge pairs probably contribute to the two-state mechanism of VS by stabilizing either the $\mathrm{C}_{\text {in }}$ or $\mathrm{C}_{\text {out }}$ state (Papazian et al. 1995). The role played by paired charges in stabilizing of the VS domain appears to be similar to that of A-like motifs conserved in MFS transporters, where these motifs stabilize their transporters in the two terminal states of a functional cycle (Zhang et al. 2015). Point mutations in such clusters, if breaking the charge pairs, will result in reduced stability of either the $C_{\text {in }}$ or $C_{\text {out }}$ state, skewing the two-state transition process. For example, mutations at the basic residues in the cytosolic half of $S 4$ cause a right shift of the $I-V$ curve of the rat $\mathrm{Na}^{+}$channel II, indicating destabilization of the $\mathrm{C}_{\text {out }}$ state. In contrast, mutations in the extracellular half of S4 result in a left shift (Stuhmer et al. 1989). Similarly, mutations at acidic residues that are involved in chargepairing often show drastic effects. For example, the D60K mutation in a cytosolic charge cluster of NaChBac shifts the $I-V$ curve $75-\mathrm{mV}$ towards depolarizing potentials (i.e., more difficult to be activated) (Zhao et al. 2004), presumably by over-stabilizing the $C_{\text {in }}$ state via electrostatic interaction with $\Delta \Psi_{\mathrm{M}}$ and by destabilizing the $\mathrm{C}_{\text {out }}$ state via disrupting charge-pairing. In addition, substituting R1 with a small side-chain residue resulted in formation of a cation channel running through the VS domain in its $\mathrm{C}_{\text {in }}$ conformation (Tombola et al. 2005b), presumably by breaking the seal formed by the charge paring in the $\mathrm{C}_{\text {in }}$ state. Moreover, folding defects from mutations that neutralize basic residues in S4 can be suppressed by neutralization of acidic residues in either S2 or S3 (Papazian et al. 1995). Of particular interest are insights gained from the charge-reversal mutations in the Drosophila Shaker $K_{\mathrm{V}}$ channel (Papazian et al. 1995; Tiwari-Woodruff et al. 2000), in which the paired, charged residues in the VS domains were mutually switched yet the mutants maintained the channel activity. Taken together, while unpaired charges are more likely to contribute to the gating-charges, the paired charges stabilize the terminal states, and alternating stabilization of both states is essential for proper functioning of the VS domain.

\section{Cooperativity between voltage-sensing domains}

As discussed above, the four VS domains encircle the central pore of the channel. An important question concerning the function of VGICs is whether the four VS domains function cooperatively. If so, a further question is what mechanism for cooperation can be envisaged that plausibly explains the observed voltage-activation relationship.

Upon depolarization and I-O transition, the number of positively charged residues exposed to the cytosol decreases, while the number of positively charged residues exposed to the extracellular space increases. Thus, for each voltage sensor, an efflux of net positive charges occurs across the focused electrical field of 
$\Delta \Psi_{\mathrm{M}}$ (or of the experimentally applied external voltage, $V$ ). At any given external voltage, the charge movement related to the I-O transition would be associated with an energy term $V \Delta Q$. Here, we re-define $\Delta Q$ as the gating-charges of a single VS "unit." Such a unit is either a fully cooperated ion channel (with four VS domains) or one independent VS domain. As noted above, $\Delta Q$ is contributed by all charged groups from the unit. Previously, the total gating-charges of the skeleton muscle $\mathrm{Na}^{+}$channel were estimated to be 3 per VS domain (or $\sim 12$ per channel) (Hirschberg et al. 1995), and for the KvAP channel, 2.4 per VS domain (Schmidt et al. 2009). Let the gating-charges per VS domain be $2.5 \mathrm{e}_{0}, \Delta \Delta \Psi_{M}$ be $50 \mathrm{mV}$, and the dielectric constant $(\varepsilon)$ for the protein be 2 . In the focused electrostatic field associated with the VS crevice (see Supplementary Fig. S2), the average electrostatic force that drives the conformational change of each VS domain is estimated to be $\sim 5 \mathrm{pN}$, which is fairly strong for a molecular event.

The Gibbs free energy of the I-O transition of the VS domain, $\Delta G_{\mathrm{VS}}$, can be described as

$$
\begin{aligned}
& \Delta G_{\mathrm{VS}}(V) \equiv G_{\text {out }}(V)-G_{\text {in }}(V)=\Delta G^{0}-V \Delta Q, \\
& \Delta \Delta G_{\mathrm{VS}} \equiv \Delta G_{\mathrm{VS}}\left(V_{1}\right)-\Delta G_{\mathrm{VS}}\left(V_{0}\right)=\Delta V \Delta Q,
\end{aligned}
$$

where $G_{\text {out }}$ and $G_{\text {in }}$ are Gibbs energies corresponding to the $\mathrm{C}_{\text {out }}$ and $\mathrm{C}_{\text {in }}$ conformations at a given voltage, $\Delta G^{0}$ represents their differential conformational energy at $V=0 \mathrm{mV}$, and $V_{0}$ and $V_{1}$ are the resting and activation voltages, respectively. This description of $\Delta G_{\mathrm{VS}}$ mirrors that of membrane tension-induced conformational change in mechanosensitive channels, with $V$ and $\Delta Q$ equivalent to membrane tension $(\sigma)$ and change of the cross-section area $(\Delta A)$, respectively (Zhang et al. 2016). Here, we follow the convention of thermodynamics according to which the negative $\Delta G$ is associated with an exergonic process. For most known VGICs, $\Delta G^{0}<0$, indicating that the $\mathrm{C}_{\text {out }}$ state is more stable at $V=0 \mathrm{mV}$ (one exception is the above-mentioned CiVSD, which assumes a $C_{\text {in }}$ state in the absence of membrane potential and has a $V_{1 / 2}$ of $+60 \mathrm{mV}$ (Li et al. 2014)). Since $\Delta G^{0}$ can be modulated by other mechanisms (e.g., ligand binding), a voltage-gated channel is often regulated by factors in addition to $\Delta \Psi_{\mathrm{M}}$. Moreover, to open the activation-gate, VS usually acquires energy from the activation cues, thus coupling $\Delta \Delta G_{\mathrm{VS}}$ with $\Delta G_{\text {gating }}$ (which includes but is not limited to $\Delta G_{\mathrm{dw}}$ ). Because of this coupling, mutations at the activationgate (e.g., those reported in Zhao et al. 2004) may dramatically shift the $I-V$ curve by affecting $\Delta G^{0}$ in Eq. 2 . Furthermore, in mammalian $\mathrm{Na}_{V}$ channels, the four homologous VS domains display distinct activation kinetics (Chanda and Bezanilla 2002). In the structure of NavPaS/5X0M, the gating-charges in different S4 helices assume different positions relative to the S1-S3 bundle (Shen et al. 2017). These observations suggest that different VS domains of the same channel may have distinct $\Delta Q$ and $\Delta G^{0}$ values.

For the purpose of our theoretical analysis, we assume that (1) the activation-gate is controlled by $n$ identical, fully independent VS units; and (2) in order to be activated by $\Delta \Delta \Psi_{\mathrm{M}}$, the channel opening requires all $n$ copies of the units to function collectively (Schmidt et al. 2009). Therefore, the open probability, $P_{\mathrm{o}}(V)$, of a depolarization-activated channel can be described as a Boltzmann distribution

$P_{0}(V)=\left[\frac{1}{1+\exp \left(\frac{\Delta G_{V S}}{R T}\right)}\right]^{n}$.

Thus,

$$
\begin{aligned}
P_{0}^{\prime}(V) & \equiv \frac{\mathrm{d} P_{0}}{\mathrm{~d} V}=\frac{n \Delta Q}{R T} \cdot P_{0}(V) \cdot \frac{1}{1+\exp \left(\frac{-\Delta G_{V S}}{R T}\right)}, \\
\frac{n \Delta Q}{F} & =\frac{2 R T}{F} \cdot P_{0}^{\prime}\left(V_{1 / 2}\right) \cdot \frac{\sqrt[n]{2}}{\sqrt[n]{2}-1} \\
& \approx 50 \mathrm{mV} \cdot P_{0}^{\prime}\left(V_{1 / 2}\right) \cdot \frac{\sqrt[n]{2}}{\sqrt[n]{2}-1}, \\
\Delta G^{0} & =V_{1 / 2} \cdot \Delta Q+R T \cdot \ln (\sqrt[n]{2}-1)
\end{aligned}
$$

where $V_{1 / 2}$ is defined as the voltage at which $P_{\mathrm{o}}(V)=1 /$ 2 (Zarrabi et al. 2010). For a hyperpolarization-activated channel, replace the $\Delta G_{\mathrm{VS}}$ with $-\Delta G_{\mathrm{VS}}$. Regarding these equations, a few points are worth discussion:

(1) $P_{\mathrm{o}}(V), P_{\mathrm{o}}{ }^{\prime}(V)$, as well as $V_{1 / 2}$ are experimentally measurable. The total gating-charges $\left(n \Delta Q / F\right.$, in $\mathrm{e}_{0}$ unit) and $\Delta G^{0}$ can be derived using Eqs. 6 and 7.

(2) The derived results depend on the model being used (e.g., whether $n$ equals 1 or 4 ). Here, the independency coefficient, $n$, is reciprocally related to the Hill coefficient (see $\S 11$ in Hill 1985). Whereas the traditional cooperative model of VGICs is equivalent to $n=1$ (Zarrabi et al. 2010), full independence between the four VS domains implies that $n=4$ (Schmidt et al. 2009).

(3) The larger are the total gating-charges $n \Delta Q / F$, the steeper is the $P_{\mathrm{o}}(V)$ curve at $V_{1 / 2}$ (i.e., a larger $\left.P_{\mathrm{o}}{ }^{\prime}\left(V_{1 / 2}\right)\right)$, corresponding to a more precise control of $\Delta \Psi_{\mathrm{M}}$ over the state transition of the VS unit.

(4) For depolarization-activated channels, $V_{1 / 2}$ is more positive than the resting $\Delta \Psi_{\mathrm{M}}$; for hyperpolarization-activated channels, $V_{1 / 2}$ is more negative than the resting $\Delta \Psi_{\mathrm{M}}$. Because of the linear relationship between $\Delta G^{0}$ and $V_{1 / 2}$, any cellular factors that modulate $\Delta G^{0}$ will affect $V_{1 / 2}$ 
too. For example, cAMP binding to the cytosolic domain of the hyperpolarization-activated HCN channel shifts $V_{1 / 2}$ to more positive values, thus easier to open the channel (Stieber et al. 2003).

For a given experimentally determined $P_{\mathrm{o}}{ }^{\prime}\left(V_{1 / 2}\right)$, the total gating-charges predicted based on the model of fully independent VS domains (i.e., $n=4$ ) would be approx. three times to that estimated based on fully cooperative gating (i.e., $n=1$ ). Therefore, it seems that the traditional, fully cooperative model is in better agreement with the structural analysis on available potential gating-charges than the fully independent model. Nevertheless, it is still possible that the four VS domains function in a partially cooperative manner (i.e., $1<n<4$ ). In case of full cooperativity, activation occurring in one VS domain will stimulate activation of other VS domains, through a currently unknown mechanism. If domain swapping were the cause of cooperativity, a non-swapped channel would be more likely to show full independency among its four VS domain. However, non-swapped HCN channels show even larger gating-charges ( $\sim 5 e_{0}$ per VS domain, estimated based on $n=1$ (Wainger et al. 2001)) than the typical $\left(\sim 2.5 e_{0}\right)$ gating-charges in swapped VGICs. This observation strongly suggests that it is highly unlikely for the four VS domains in a HCN channel to act in a fully independent manner, as it would require $\sim 15-e_{0}$ gating-charges per VS domain, a prediction unsupported by structural data.

On the basis of the above discussion, we propose a mechanistic model for the cooperativity in a canonical depolarization-activated ion channel. Since all four VS domains are linked to the activation-gate, their $\Delta \Delta G_{\mathrm{VS}}$ terms are coupled with each other through $\Delta G_{\text {gating }}$ of the activation-gate. On the one hand, in the absence of compression force from the VS domains, the central pore of the channel is presumably present in its open state (i.e., $\Delta G_{\text {gating }} \equiv G_{\text {open }}-G_{\text {close }}<0$ ). Such an open state is probably stabilized by the in vivo membrane environment, and thus has not been observed in most of the reported crystal structures. On the other hand, the closed state of the central pore is maintained by compression forces from all four VS domains in their $\mathrm{C}_{\text {in }}$ state. Therefore, it appears that being in the $\mathrm{C}_{\text {in }}$ state for one of the four VS domains is a sufficient (but not necessary) condition for keeping the activation-gate close. In other words, being in the $\mathrm{C}_{\text {out }}$ state for all four VS domains is a necessary (but not sufficient) condition for keeping the activation-gate open. In energetic terms, under the condition of polarized $\Delta \Psi_{\mathrm{M}}, \Delta G_{\text {gating }}$ of the activation-gate is canceled out by $\Delta G_{\mathrm{VS}}$ contributed by the surrounding VS domains. Upon the I-O transition of one VS domain, the compression on the central pore is partially removed. Consequently, the force to maintain the closed state of the activation-gate is likely reshuffled among the remaining VS domains that are still in contact with the activation-gate. As a result, these remaining VM domains become overloaded, because they themselves are also in the margin of $V_{1 / 2}$. This increased force, which originated from the negative $\Delta G_{\text {gating }}$ and is shared by the VS domains remaining in $\mathrm{C}_{\text {in }}$ states, will facilitate further I-O transition in other VS domains, increasing their probability to be activated (with a decreased $\Delta G^{0}$ ). In this domino manner, a cooperative activation of all four VS domains as well as the central pore is realized. Therefore, on the basis of the charged residues available in the VS domain, we propose that the four encircling VS domains in the tetrameric VGIC use a cooperative mechanism to activate the central pore.

\section{Coupling between the VS domains and the central pore}

The voltage-sensing mechanisms are similar in both depolarization- and hyperpolarization-activated channels, performing the same I-O transition upon $\Delta \Psi_{\mathrm{M}^{-}}$ depolarization. However, for the VS domain of a depolarization-activated channel, $C_{\text {in }}$ corresponds to the resting state of the channel, and $\mathrm{C}_{\text {out }}$ corresponds to the activated state; for hyperpolarization-activated channels, the situation is reversed. Thus, for depolarizationactivated channels the I-O transition is associated with the gate opening, whereas for hyperpolarizationactivated channels, the same I-O transition is associated with the gate closing. In either case, part of the activation energy from the VS domain is coupled to the activation-gate, either removing energy barrier or compensating the energy cost of gate opening.

The question then arises as to how the VS domains communicate with the activation-gate. Two types of packing, swapped and non-swapped, have been found between the VS and pore-domains. While the swapped assembly is more commonly observed, the nonswapped assemblies are currently only found in the two crystal structures of Eag1/5K7L (Whicher and MacKinnon 2016) and HCN1/5U60 (Lee and MacKinnon 2017). We first discuss the swapped assembly, in which the VS domain from each subunit packs sequentially against the pore domain of its neighboring subunit. The VS domain contacts the central pore through multiple patches of interaction, including an inter-subunit contact between the S1-S2 loop and C-terminus of S5' (where the prime indicates being from a neighboring subunit); an extensive inter-subunit contact between anti-paralleled S4 and S5'; and an 
intra-subunit, VS-pore connection through the amphipathic linker helix, S4-S5 (Long et al. 2005; Payandeh et al. 2011). During the conformational transition of the VS domain, these multiple contacts change their relative arrangement, transducing $\Delta G_{\mathrm{VS}}$ from the VS domain to the central pore. For example, while in the $C_{\text {in }}$ state of the structure of $\mathrm{Na}_{\mathrm{V}} \mathrm{Ab}$, a salt-bridge bond is presumably formed between $\mathrm{K} 35^{\mathrm{S} 1}$ and $\mathrm{E} 154^{\mathrm{S5}}$, this bond is lost in the $C_{\text {out }}$ structure (3RVY) (Payandeh et al. 2011). Disruption of these inter- and intra-subunit interactions is likely to perturb the communication between the VS domains and the central pore. Intriguingly, a number of disease-related mutations in human $\mathrm{Na}_{\mathrm{V}}$ channels have been mapped to the interface between S4 and S5' (Shen et al. 2017). Experimentally, mutations in the S1-S2 loop region were found to affect the activation rate (Stieber et al. 2003). In addition, the signal transduction and energy coupling are believed to be relayed by the amphipathic S4-S5 helix, which connects S4 of the VS domain to $\mathrm{S} 5$ of the pore domain. This inter-domain connection is, however, likely to be only part of the function of the S4-S5 helix. In general, amphipathic helices play important roles in restricting conformational changes of transmembrane helices (Zhang et al. 2017b). As mentioned above, in 6TM channels, the S4S5 helix anchors the cytosolic termini of both helices S4 and S5 to the intracellular surface of the lipid bilayer, converting "vertical" forces (e.g., those associated with $\Delta \Psi_{\mathrm{M}}$ and hydrophobic mismatch) perpendicular to the membrane to "horizontal" movements of the helix termini parallel to the membrane surface. Therefore, this anchoring effect of the S4-S5 helix is an important component of the sliding-rocking model of VS. More importantly, the horizontal movement of the C-terminus of S4 adjusts the size of a constricting ring formed by the four S4-S5 amphipathic helices surrounding the activation-gate, and thus controls the gating process. Firstly, during the 0-I transition, the movements of both S4 and S4-S5 helices result in a change of the angle between them, and presumably tightens the constricting ring of the activation-gate, thus forcing the channel to close (Payandeh et al. 2011). The communication between VS and the central pore is reciprocal. For instance, preventing the central pore from entering its close state by using a blocker molecule has been shown to significantly slower the rate of the O-I transition of VS domains in the Shaker $K_{\mathrm{V}}$ channel (Tombola et al. 2005b). Secondly, in its $C_{\text {out }}$ state, the VS domain loosens the constricting ring and releases the compression on the activation-gate, thus allowing the gate to open. Similar mechanism of coupling between S4 and the pore domain has been proposed based on the structural analysis on the EeNa ${ }_{v} 1.4$ channel (Yan et al. 2017). Non- swapped, depolarization-activated channels (e.g., Eag1) are likely to use a similar mechanism to gate the channel, albeit in this case the interactions are all intra-subunit. Moreover, in the 3D structure of hyperpolarization-activated channel HCN1/5U60, both the amphipathic helix S4-S5 and the constricting ring are absent. Instead, S4 and S5 are connected through a short loop. For such cases we hypothesize that, during the $0-\mathrm{I}$ transition (i.e., activation by hyperpolarization), movement of S4 displaces S5 from the activation-gate and removes constrain of S5 on S6, thus opening the gate. In all cases, the movement of the cytosolic terminus of S4 relative to the central pore seems to function as a trigger to activate the gate of the central pore.

The VS domain may lose its coupling with the pore domain under certain in vitro conditions, as exemplified in the crystal structures of $\mathrm{Na}_{V} \mathrm{Ab} / 3 \mathrm{RVY}$ and $\mathrm{Na}_{\mathrm{V}} \mathrm{Rh} /$ 4DXW (Zhang et al. 2012). In these structures, the VS domain appears in the active $\mathrm{C}_{\text {out }}$ state, but the activation-gate remains closed. This apparent inconsistency presumably results from the response of certain channels to the loss of the native membrane environment (including both $\Delta \Psi_{\mathrm{M}}$ and proper hydrophobic interaction). In addition, in the Eag1 channel (a depolarizationactivated non-swapped channel), the VS domain and pore domain can be decoupled by calmodulin (Whicher and MacKinnon 2016). Binding of calmodulin to the intracellular domains directly compresses the C-termini of $\mathrm{S} 6$ helices, and thus the gate becomes non-responsive to voltage-controlled S4 movement. Interestingly, certain Pro-substitution mutations in the pore-lining helix S6 of the depolarization-activated tetrameric $\mathrm{Na}^{+}$channel, NaChBac, result in hyperpolarization-activated variants (Zhao et al. 2004), i.e., reversal of the polarity of the voltage-dependent activation. This observation suggests that the coupling between the VS domain and activationgate can be modulated by subtle structural changes at their interface.

\section{INACTIVATION OF THE $\mathrm{Na}^{+}$CHANNELS}

Inactivation is one of the characteristic phenomena of all ion channels, and can be explained based on the above structural and thermodynamic discussions. While a transport rate of $10^{7}$ ions per second per channel might be achieved in electrophysiology experiments, such a high rate of continuing transport is unsustainable for any living cell. Under physiological conditions, the transport in depolarization-activated $\mathrm{K}^{+}$channels is mainly driven by the Nernst potential of $\mathrm{K}^{+}$ions but against $\Delta \Psi_{\mathrm{M}}$. Thus, cells are protected from depletion by negative feedback of these channels. In contrast, 
because their ion flows reduce $\Delta \Psi_{\mathrm{M}}$, depolarizationactivated $\mathrm{Na}^{+}$(and $\mathrm{Ca}^{2+}$ ) channels usually function in a positive feedback manner. Therefore, $\mathrm{a} \mathrm{Na}^{+}$channel must be equipped with an inactivation mechanism to quickly auto-deactivate itself after activation. More specifically, $\mathrm{Na}^{+}$channels from both eukaryotic and prokaryotic cells undergo a slow inactivation, with a time constant in the range from tens to hundreds of millisecond (Zarrabi et al. 2010). In addition to this mechanism, eukaryotic $\mathrm{Na}^{+}$channels often exhibit fast inactivation (with a time constant in the millisecond range) (Bagneris et al. 2015), which is so fast that an ensemble of the channels has not reached its "true" maximum current $\left(I_{\max }\right)$ before all channels are closed (Schmidt et al. 2009). In addition, certain types of $\mathrm{K}^{+}$ channels (e.g., the Shaker $K_{\mathrm{V}}$ channels) and hyperpolarization-activated channels (e.g., HCNs) also exhibit inactivation in the presence of prolonged activation cues (Hoshi et al. 1991; Rothberg et al. 2002). Many mutational inherited diseases that target VGICs are shown to impair the inactivation function (Catterall 2010).

Several hypotheses on the mechanisms of inactivation for ion channels have been postulated, including collapse of the selectivity-filter, blocking of the activation-gate by tethered polypeptides, reshaping of the activation-gate, and allosteric blocking (Bagneris et al. 2015; Cuello et al. 2010b; Payandeh et al. 2012; Yan et al. 2017). While each model is supported by experimental data and may function in certain types of channels, the exact type of energetic driving forces for such inactivation and for later recovery from the inactive state remains to be clarified. In eukaryotic $\mathrm{Na}^{+}$ channels, fast inactivation after opening of the gate is achieved through releasing a tethered polypeptide (i.e., the blocker) from an idle state and/or exposing a binding site for the blocker, thus leading to subsequent gate blockage. Such blockage is reversed immediately after the channel is reset back to its resting condition. For example, a cytoplasmic inactivation lid located between the third and fourth homologous 6TM repeats of the mammalian $\mathrm{Na}^{+}$channel confers fast inactivation through a hinged-lid mechanism (Stuhmer et al. 1989). In addition, the Shaker $K_{\mathrm{V}}$ channel was shown to be plugged by its $\mathrm{N}$-terminal polypeptide which acts as a blocker of the ion permeation pathway. If the $\mathrm{N}$-terminus is truncated, inactivation is slowed down (Hoshi et al. 1990). This so-called N-type fast inactivation is thought to permit the channel to be available for quick re-activation, for example in the presence of high-frequency activation pulses (Hoshi et al. 1990). In comparison, slow inactivation (also called C-type inactivation) is affected by structural perturbations in the C-terminal region (including the S6 helix). However,
N-type activation and C-type inactivation are not mutually exclusive, and have been shown to occur simultaneously in a number of channels (Hoshi et al. 1991).

Unlike fast inactivation, which is usually associated with channel-specific mechanisms, slow inactivation is more likely to be used as a common mechanism across different channels. Here, we will discuss $\mathrm{Na}^{+}$channels lacking fast inactivation as an example to illustrate such a common mechanism. After prolonged activation under a persistent activation cue, the $\mathrm{Na}^{+}$channel gradually loses all of its conductivity. Under the resting condition, the deactivated channel takes certain time to return to the state capable to become fully activated again. This relaxation process is called recovery. In addition, after pre-treating the channel with a mild activation cue, the channel shows a reduced " $I_{\max }$ " in response to a fullstrength activation cue; this phenomenon is called desensitization. Interestingly, raising the concentration of substrate ions increases the recovery rate from slow inactivation (Townsend and Horn 1997), suggesting that whatever blocks the gate during slow inactivation competes with the substrate ions. Single-channel recordings (e.g., in Hoshi et al. 1991) showed that the slow inactivation is associated with a suddenly reduced opening frequency but not a gradually lowered current (or $g_{\text {sc }}$ ) for a single channel.

It has long been proposed that blockers acting in channel inactivation may function through either hydrophobic or hydrophilic pathways (Hille 1977). Between these two routes, the hydrophobic path is more likely to be associated with slow inactivation as this path exhibits a slower diffusion rate. Here, we propose a model of reaction kinetics for the general lipid-infiltration-mediated mechanism in charge of the slow inactivation in $\mathrm{Na}^{+}$channels (Fig. 4). In our model, the surrounding lipid molecules behave as reversible inhibitors (blockers) and penetrate into the activationgate through interfaces between the subunits, thus deactivating the opened channel by blocking the activation-gate. This model is reminiscent of a model proposed before the first ion channel was cloned (Hille 1977). However, in comparison to the previous model, we provide a physical explanation for the process of slow inactivation. A comparable mechanism has also been used to explain the adaptation phenomenon in mechanosensitivity channels of small-conductance (MscS) (Zhang et al. 2016). Curiously, the slow inactivation observed in these two types of channels appears to follow similar pathways: both become inactivated only after the gate is opened; both are sensitive to mild activation cues; and both require considerable recovery time before restoring their ability to be activated again. 
A

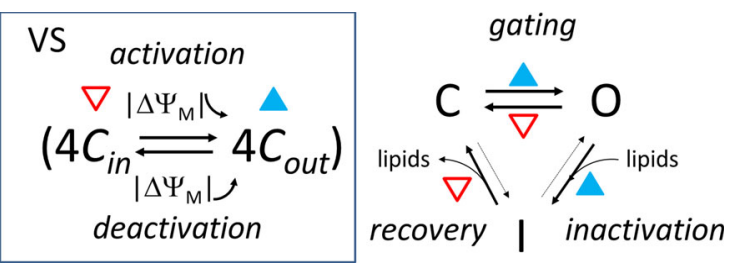

B

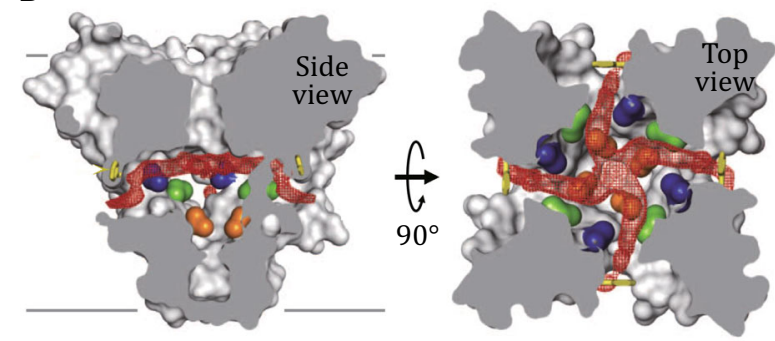

Fig. 4 Putative mechanism of the slow inactivation of ion channels. A Kinetic model of the gating process. Represented in the box, the VS domain has a $\mathrm{C}_{\text {in }}$ state (open triangle) and a $\mathrm{C}_{\text {out }}$ state (filled triangle). On the right side, the activation-gate has a closed (C) state, open (O) state, and an inactivated (I) state. The state of the VS domain is coupled to that of the activation-gate. The thickness of the arrows indicates the rate of the corresponding reaction. B Fenestrations on the wall of the central pore in the closed state of $\mathrm{Na}_{\mathrm{V}} \mathrm{Ab} / 3 \mathrm{RVY}$. Red electron densities correspond to lipid penetration paths. These paths are presumably enlarged upon the activation-gate is switched to the open state, thus allowing more rapid infiltration of lipid molecules into the gate pore. This panel is adapted from this figure in Payandeh et al. (2011)

In addition, the inactivation phenomenon of ion channels is qualitatively similar to reversible inhibition by certain toxic drugs (Schmidt et al. 2009). In fact, drug blocking and inactivation appear to enhance each other (Hille 1977). Both types of inhibition can be explained by structural changes at the activation-gate upon activation. In particular, the pore-lining S6 helices become loosely packed with each other once the gate is opened, allowing surrounding lipid molecules to infiltrate slowly into the gate pore. Such penetration will result in reduced pore size and increased hydrophobicity of the gate pore, thus decreasing the open probability of the gate by virtual of the dry/wet two-state mechanism in which a constant $g_{\mathrm{sc}}$ is assumed for the single channel. In contrast to the sponge-like behavior observed for the open pore in lipid absorption, the closed pore slowly squeezes out the plugging lipid molecules in the recovery process upon withdrawing the activation cue. In addition, the diffusion rate of lipid molecules into the activation-gate is likely to be positively correlated with the size of the gate. Thus, the rate of slow inactivation depends on activation cues. In particular, the lipids may infiltrate into the activation-gate even before it enters its wet state. Therefore, pre-activation with mild activation cues results in a partial filling of the gate pore (or a fraction of the channel ensemble) with lipid molecules, thus reducing the probability of gate opening. Together, these arguments should explain how desensitization is achieved by the lipid-infiltration mechanisms.

In agreement with our hypothesis, in the closed-state structure of $\mathrm{Na}_{\mathrm{V}} \mathrm{Ab} / 3 \mathrm{RVY}$, four lateral portals (termed fenestrations) are found on the wall of the central cavity, connecting the lipid bilayer to the interior of the central pore (Payandeh et al. 2011). These fenestrations were unexpectedly penetrated by fatty acyl chains that extended into the central cavity (Fig. 4B). The portals are suspected to serve as the entries for lipophilic inhibitors, including local anesthetics. Importantly, these portals become enlarged in the open state of the activation-gate (McCusker et al. 2012), presumably allowing more rapid penetration of lipid molecules into the central pore. Similarly, in the crystal structure of $\mathrm{Na}_{\mathrm{v}} \mathrm{Rh} / 4 \mathrm{DXW}$, a lipid/detergent molecule is observed in the central cavity, and in the crystal structure of $\mathrm{Na}_{\mathrm{V}} 1.4$ / 5XSY from electric eel $\left(E^{2} \mathrm{Na}_{\mathrm{V}} 1.4\right)$, the activation-gate was found to be "kept open" by a detergent-like molecule inside the gate (Yan et al. 2017). Moreover, changing the hydrophobicity and saturation properties of lipid molecules of the membrane bilayer also affects the rate of slow inactivation (Schmidt et al. 2009). Also in line with our hypothesis of lipid-infiltration-mediated inactivation, mutations in the middle of S6 near the fenestration (e.g., I1575A in repeat-IV of $\mathrm{rNa}_{\mathrm{V}} 1.4$ ) were shown to increase the rates of both inactivation and recovery (Zarrabi et al. 2010), probably by virtual of reducing kinetic barrier for lipid infiltration. A singlepoint mutation at a conserved Asn residue in S6 alters slow inactivation in $\mathrm{Na}_{\mathrm{V}}$ channels (Wang and Wang 1997). In contrast to our hypothesis, the model of collapsed selectivity-filter for slow inactivation would predict gradually reduced $g_{\mathrm{sc}}$ value in the single-channel recording, which strongly contradicts presently available experimental observations (Cuello et al. 2010a).

The pore-lining $\mathrm{S} 6$ helices of $\mathrm{Na}^{+}$channels usually maintain a degree of helix bundle crossing that is lower than those found in inactivation-resisting $\mathrm{K}^{+}$channels. In the open state, the C-terminal ends of S6 helices become more flexible than the closed state (e.g., in $\mathrm{Na}_{\mathrm{V}} \mathrm{Ms}$ /3ZJZ), allowing formation of dynamic portals between subunits. In addition, subunits of bacterial $\mathrm{Na}^{+}$ channels often contain C-terminal domains as an extension of S6, which are further tetramerized in the cytosol. In crystal structures of closed gates (e.g., in

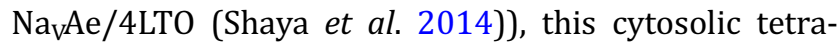
meric region, in particular the linkers between S6 helices and C-terminal helix bundle, is well ordered; 
however, it becomes (partially) disordered in the open state, presumably permitting formation of lateral portals on the wall of the activation-gate. The rate of inactivation in $\mathrm{Na}_{V} \mathrm{Ms}$ is also positively related to the presence of the flexible linker and, in particular, of three consecutive acidic residues in this region (Bagneris et al. 2013). Presumably, the repulsion between these concentrated $(3 \times 4)$ acidic residues maintains the lateral portals of the gate, thus permitting inactivation to proceed. Another channel whose crystal structure (3EFD) has been reported to show straight (rather than crossing) pore-lining helices is the full-length channel KcsA of S. lividans. If its cytosolic C-terminal four-helix bundle region is stabilized following Fab binding, the inactivation rate is reduced (Uysal et al. 2009). In contrast, in inactivation-resisting voltage-gated $\mathrm{K}^{+}$channels, the C-terminal regions of S6 helices are more curved and tilted (e.g., in the crystal structure of Kv1.2/2A79), forming a highly crossing, right-handed bundle. This more twisted helix bundle is likely to resist lipid penetration, in a manner similarly to the MscL-type of mechanosensitivity channels (Zhang et al. 2016). In addition, subunits of $\mathrm{K}^{+}$channels often contain an $\mathrm{N}$-terminal domain which is tetramerized in cytosol "underneath" the activation-gate (e.g., in Kv1.2/2A79), presumably constricting the overall flexibility of the channel tetramer. These structural features may contribute to the lack of inactivation in certain $\mathrm{K}^{+}$channels. Moreover, the acidic residue introduced by the abovementioned K1237E mutation in the filter region of repeat-IV of $\mathrm{rNa}_{\mathrm{V}} 1.4$ (Todt et al. 1999) is subjected to an extra electrostatic force, compared with the wild-type channel. This electrical force shifts the central pore towards the extracellular direction. Together with the hydrophobic mismatch, this outward force is likely to make the pore-lining helices of the activation-gate more compact, thus reducing the penetration probability of surrounding lipid molecules and resulting in ultra-slow inactivation (Todt et al. 1999). Taken together, dynamic inter-subunit portals between $\mathrm{S} 6$ helices in $\mathrm{Na}_{\mathrm{V}}$ channels are likely to serve as the paths for lipid molecules to penetrate into and exit from the central pore during inactivation and recovery, respectively.

\section{CONCLUDING REMARKS}

The microenvironment provided by the cellular membrane, including lipid bilayer and the carried electrostatic membrane potential, plays essential roles in ensuring proper functioning of ion channels. The thermodynamic interactions between channels and their membrane environments are keys to understanding of the molecular mechanisms of channel operation. However, by emphasizing the common mechanisms of VGICs, we do not suggest that individual details and mechanisms are of lesser importance to the overall knowledge on ion channels. Our message distilled here was formed through appropriate abstraction, and is based on the assumption that all functions of membrane proteins result from generally applicable physical principles. We anticipate that meaningful combination of quantitative thermodynamic consideration with detailed structural studies as well as biochemical and functional analyses will improve our understanding of the general principle of ion channel operation as well as the detailed mechanisms of individual molecular targets.

$\begin{array}{ll}\text { Abbreviations } \\ \mathrm{HCN} & \begin{array}{l}\text { Hyperpolarization-activated cyclic nucleotide- } \\ \text { gated channel }\end{array} \\ \mathrm{Kcs} & \begin{array}{l}\mathrm{K}^{+} \text {channel from } \text { Streptomyces lividans } \\ \text { Koltage-gated } \mathrm{K}^{+} \text {channel from Aeropyrum } \\ \text { pernix }\end{array} \\ \mathrm{Na}_{\mathrm{V}} \mathrm{Ab} & \begin{array}{l}\text { Voltage-gated } \mathrm{Na}^{+} \text {channel from Arcobacter } \\ \text { butzleri }\end{array} \\ \mathrm{Na}_{\mathrm{V}} \mathrm{Ae} & \begin{array}{l}\text { Voltage-gated } \mathrm{Na}^{+} \text {channel from } \\ \text { Alkalilimnicola ehrlichei }\end{array} \\ \mathrm{Na}_{\mathrm{V}} \mathrm{Ms} & \begin{array}{l}\text { Voltage-gated } \mathrm{Na}^{+} \text {channel from } \\ \text { Magnetococcus spirillium }\end{array} \\ \mathrm{Na}_{\mathrm{V}} \mathrm{Rh} & \begin{array}{l}\text { Voltage-gated } \mathrm{Na}^{+} \text {channel from Rickettsiales } \\ \text { sp. }\end{array} \\ \mathrm{TM} & \begin{array}{l}\text { Transmembrane (helix) } \\ \text { Two-pore channel }\end{array} \\ \mathrm{TPC} & \begin{array}{l}\text { Voltage-gated ion channel } \\ \text { VGIC }\end{array} \\ \mathrm{VS} & \text { Voltage sensor }\end{array}$

Acknowledgement The authors thank Dr. Torsten Juelich (Peking University, China) for linguistic assistance during the preparation of this manuscript. This work was supported by the CAS Strategic Priority Research Program (XDB08020301), the Ministry of Science and Technology of China (2015CB910104 and 2016DDJ1ZZ17), and the National Natural Science Foundation of China (31470745).

\section{Compliance with Ethical Standards}

Conflict of interest Xuejun C. Zhang, Hanting Yang, Zhenfeng Liu, and Fei Sun declare that they have no conflict of interest.

Human and animal rights and informed consent This article does not contain any studies with human or animal subjects performed by any of the authors.

Open Access This article is distributed under the terms of the Creative Commons Attribution 4.0 International License (http:// creativecommons.org/licenses/by/4.0/), which permits 
unrestricted use, distribution, and reproduction in any medium, provided you give appropriate credit to the original author(s) and the source, provide a link to the Creative Commons license, and indicate if changes were made.

\section{References}

Anishkin A, Sukharev S (2004) Water dynamics and dewetting transitions in the small mechanosensitive channel MscS. Biophys J 86:2883-2895

Anishkin A, Akitake B, Kamaraju K, Chiang CS, Sukharev S (2010) Hydration properties of mechanosensitive channel pores define the energetics of gating. J Phys 22:454120

Armstrong CM, Bezanilla F (1973) Currents related to movement of the gating particles of the sodium channels. Nature 242:459-461

Bagneris C, Decaen PG, Hall BA, Naylor CE, Clapham DE, Kay CW, Wallace BA (2013) Role of the C-terminal domain in the structure and function of tetrameric sodium channels. Nat Commun 4:2465

Bagneris C, Naylor CE, McCusker EC, Wallace BA (2015) Structural model of the open-closed-inactivated cycle of prokaryotic voltage-gated sodium channels. J Gen Physiol 145:5-16

Benedek GB, Villars F (2000) Physics, with illustrative examples from medicine and biology (electricity and magmetism), vol 3, 2nd edn. AIP Press, New York

Catterall WA (2000) From ionic currents to molecular mechanisms: the structure and function of voltage-gated sodium channels. Neuron 26:13-25

Catterall WA (2010) Ion channel voltage sensors: structure, function, and pathophysiology. Neuron 67:915-928

Cha A, Bezanilla F (1997) Characterizing voltage-dependent conformational changes in the Shaker $\mathrm{K}^{+}$channel with fluorescence. Neuron 19:1127-1140

Chanda B, Bezanilla F (2002) Tracking voltage-dependent conformational changes in skeletal muscle sodium channel during activation. J Gen Physiol 120:629-645

Chanda B, Asamoah OK, Blunck R, Roux B, Bezanilla F (2005) Gating charge displacement in voltage-gated ion channels involves limited transmembrane movement. Nature 436:852-856

Cuello LG, Jogini V, Cortes DM, Pan AC, Gagnon DG, Dalmas O, Cordero-Morales JF, Chakrapani S, Roux B, Perozo E (2010a) Structural basis for the coupling between activation and inactivation gates in $\mathrm{K}(+)$ channels. Nature 466:272-275

Cuello LG, Jogini V, Cortes DM, Perozo E (2010b) Structural mechanism of C-type inactivation in $\mathrm{K}(+)$ channels. Nature 466:203-208

DeCaen PG, Yarov-Yarovoy V, Sharp EM, Scheuer T, Catterall WA (2009) Sequential formation of ion pairs during activation of a sodium channel voltage sensor. Proc Natl Acad Sci USA 106:22498-22503

Deisseroth K, Hegemann P (2017) The form and function of channelrhodopsin. Science 357:eaan5544

des Georges A, Clarke OB, Zalk R, Yuan Q, Condon KJ, Grassucci RA, Hendrickson WA, Marks AR, Frank J (2016) Structural basis for gating and activation of RyR1. Cell 167:145-157

Doyle DA, Morais Cabral J, Pfuetzner RA, Kuo A, Gulbis JM, Cohen SL, Chait BT, MacKinnon R (1998) The structure of the potassium channel: molecular basis of $\mathrm{K}^{+}$conduction and selectivity. Science 280:69-77
Gonzalez C, Rosenman E, Bezanilla F, Alvarez O, Latorre R (2000) Modulation of the Shaker $\mathrm{K}(+)$ channel gating kinetics by the S3-S4 linker. J Gen Physiol 115:193-208

Guo J, Zeng W, Chen Q Lee C, Chen L, Yang Y, Cang C, Ren D, Jiang Y (2016) Structure of the voltage-gated two-pore channel TPC1 from Arabidopsis thaliana. Nature 531:196-201

Gutman GA, Chandy KG, Grissmer S, Lazdunski M, McKinnon D, Pardo LA, Robertson GA, Rudy B, Sanguinetti MC, Stuhmer W, Wang X (2005) International Union of Pharmacology. LIII. Nomenclature and molecular relationships of voltage-gated potassium channels. Pharmacol Rev 57:473-508

Haswell ES, Phillips R, Rees DC (2011) Mechanosensitive channels: what can they do and how do they do it? Structure 19:1356-1369

Hill T (1985) Cooperativity theory in biochentistry: steady-state and equilibrium systems. Springer, New York Inc

Hille B (1977) Local anesthetics: hydrophilic and hydrophobic pathways for the drug-receptor reaction. J Gen Physiol 69:497-515

Hirschberg B, Rovner A, Lieberman M, Patlak J (1995) Transfer of twelve charges is needed to open skeletal muscle $\mathrm{Na}^{+}$ channels. J Gen Physiol 106:1053-1068

Hosaka T, Okazaki M, Kimura-Someya T, Ishizuka-Katsura Y, Ito K, Yokoyama S, Dodo K, Sodeoka M, Shirouzu M (2017) Crystal structural characterization reveals novel oligomeric interactions of human voltage-dependent anion channel 1. Protein Sci 26:1749-1758

Hoshi T, Zagotta WN, Aldrich RW (1990) Biophysical and molecular mechanisms of Shaker potassium channel inactivation. Science 250:533-538

Hoshi T, Zagotta WN, Aldrich RW (1991) Two types of inactivation in Shaker $\mathrm{K}^{+}$channels: effects of alterations in the carboxyterminal region. Neuron 7:547-556

Howarth C, Gleeson P, Attwell D (2012) Updated energy budgets for neural computation in the neocortex and cerebellum. J Cereb Blood Flow Metab 32:1222-1232

Islas LD, Sigworth FJ (2001) Electrostatics and the gating pore of Shaker potassium channels. J Gen Physiol 117:69-89

Jackson HA, Marshall CR, Accili EA (2007) Evolution and structural diversification of hyperpolarization-activated cyclic nucleotide-gated channel genes. Physiol Genomics 29:231-245

Jan LY, Jan YN (1989) Voltage-sensitive ion channels. Cell $56: 13-25$

Jardetzky 0 (1966) Simple allosteric model for membrane pumps. Nature 211:969-970

Kintzer AF, Stroud RM (2016) Structure, inhibition and regulation of two-pore channel TPC1 from Arabidopsis thaliana. Nature 531:258-262

Kintzer AF, Stroud RM (2017) On the structure and mechanism of two-pore channels. FEBS J 285:233-243

Lee CH, MacKinnon R (2017) Structures of the human HCN1 hyperpolarization-activated channel. Cell 168(111-120):e111

Li Q, Wanderling S, Paduch M, Medovoy D, Singharoy A, McGreevy R, Villalba-Galea CA, Hulse RE, Roux B, Schulten K, Perozo E (2014) Structural mechanism of voltage-dependent gating in an isolated voltage-sensing domain. Nat Struct Mol Biol 21:244-252

Liu F, Zhang Z, Csanady L, Gadsby DC, Chen J (2017) Molecular structure of the human CFTR ion channel. Cell 169(85-95):e88

Long SB, Campbell EB, Mackinnon R (2005) Crystal structure of a mammalian voltage-dependent Shaker family $\mathrm{K}^{+}$channel. Science 309:897-903 
Mannuzzu LM, Moronne MM, Isacoff EY (1996) Direct physical measure of conformational rearrangement underlying potassium channel gating. Science 271:213-216

McCoy JG, Nimigean CM (2012) Structural correlates of selectivity and inactivation in potassium channels. Biochim Biophys Acta 1818:272-285

McCoy JG, Ren Z, Stanevich V, Lee J, Mitra S, Levin EJ, Poget S, Quick M, Im W, Zhou M (2016) The structure of a sugar transporter of the glucose EIIC superfamily provides insight into the elevator mechanism of membrane transport. Structure 24:956-964

McCusker EC, Bagneris C, Naylor CE, Cole AR, D’Avanzo N, Nichols CG, Wallace BA (2012) Structure of a bacterial voltage-gated sodium channel pore reveals mechanisms of opening and closing. Nat Commun 3:1102

Memon T, Chase K, Leavitt LS, Olivera BM, Teichert RW (2017) TRPA1 expression levels and excitability brake by KV channels influence cold sensitivity of TRPA1-expressing neurons. Neuroscience 353:76-86

Miller PS, Aricescu AR (2014) Crystal structure of a human GABAA receptor. Nature 512:270-275

Novella Romanelli M, Sartiani L, Masi A, Mannaioni G, Manetti D, Mugelli A, Cerbai E (2016) HCN channels modulators: the need for selectivity. Curr Top Med Chem 16:1764-1791

Papazian DM, Shao XM, Seoh SA, Mock AF, Huang Y, Wainstock DH (1995) Electrostatic interactions of S4 voltage sensor in Shaker $\mathrm{K}^{+}$channel. Neuron 14:1293-1301

Payandeh J, Scheuer T, Zheng N, Catterall WA (2011) The crystal structure of a voltage-gated sodium channel. Nature 475:353-358

Payandeh J, Gamal El-Din TM, Scheuer T, Zheng N, Catterall WA (2012) Crystal structure of a voltage-gated sodium channel in two potentially inactivated states. Nature 486:135-139

Phillips R, Ursell T, Wiggins P, Sens P (2009) Emerging roles for lipids in shaping membrane-protein function. Nature 459:379-385

Pohorille A, Schweighofer K, Wilson MA (2005) The origin and early evolution of membrane channels. Astrobiology 5:1-17

Purves D, Augustine G, Fitzpatrick D, Katz L, LaMantia A, McNamara J, Williams S (2001) Voltage-gated ion channels. In: Neuroscience. Sinauer Associates, Sunderland

Qian H, Kou SC (2014) Statistics and related topics in singlemolecule biophysics. Annu Rev Stat Appl 1:465-492

Ren D, Navarro B, Xu H, Yue L, Shi Q, Clapham DE (2001) A prokaryotic voltage-gated sodium channel. Science 294:2372-2375

Rothberg BS, Shin KS, Phale PS, Yellen G (2002) Voltage-controlled gating at the intracellular entrance to a hyperpolarizationactivated cation channel. J Gen Physiol 119:83-91

Roux B (1997) Influence of the membrane potential on the free energy of an intrinsic protein. Biophys J 73:2980-2989

Ruta V, Chen J, MacKinnon R (2005) Calibrated measurement of gating-charge arginine displacement in the KvAP voltagedependent $\mathrm{K}^{+}$channel. Cell 123:463-475

Saier MH Jr (2016) Transport protein evolution deduced from analysis of sequence, topology and structure. Curr Opin Struct Biol 38:9-17

Sakata S, Jinno Y, Kawanabe A, Okamura Y (2016) Voltagedependent motion of the catalytic region of voltage-sensing phosphatase monitored by a fluorescent amino acid. Proc Natl Acad Sci USA 113:7521-7526

Schmidt D, Cross SR, MacKinnon R (2009) A gating model for the archeal voltage-dependent $\mathrm{K}(+)$ channel KvAP in DPhPC and POPE:POPG decane lipid bilayers. J Mol Biol 390:902-912

Shaya D, Findeisen F, Abderemane-Ali F, Arrigoni C, Wong S, Nurva SR, Loussouarn G, Minor DL Jr (2014) Structure of a prokaryotic sodium channel pore reveals essential gating elements and an outer ion binding site common to eukaryotic channels. J Mol Biol 426:467-483

She J, Guo J, Chen Q Zeng W, Jiang Y, Bai XC (2018) Structural insights into the voltage and phospholipid activation of the mammalian TPC1 channel. Nature 556:130-134

Shen H, Zhou Q, Pan X, Li Z, Wu J, Yan N (2017) Structure of a eukaryotic voltage-gated sodium channel at near-atomic resolution. Science 355:eaal4326

Smith E, Morowitz HJ (2016) The origin and nature of life on Earth: the emergence of the fourth geosphere. Cambridge University Press, New York

Starace DM, Bezanilla F (2001) Histidine scanning mutagenesis of basic residues of the S4 segment of the shaker $\mathrm{K}^{+}$channel. J Gen Physiol 117:469-490

Stieber J, Thomer A, Much B, Schneider A, Biel M, Hofmann F (2003) Molecular basis for the different activation kinetics of the pacemaker channels HCN2 and HCN4. J Biol Chem 278:33672-33680

Stuhmer W, Conti F, Suzuki H, Wang XD, Noda M, Yahagi N, Kubo H, Numa S (1989) Structural parts involved in activation and inactivation of the sodium channel. Nature 339:597-603

Tao X, Lee A, Limapichat W, Dougherty DA, MacKinnon R (2010) A gating charge transfer center in voltage sensors. Science 328:67-73

Thompson AN, Posson DJ, Parsa PV, Nimigean CM (2008) Molecular mechanism of $\mathrm{pH}$ sensing in KcsA potassium channels. Proc Natl Acad Sci USA 105:6900-6905

Tiwari-Woodruff SK, Lin MA, Schulteis CT, Papazian DM (2000) Voltage-dependent structural interactions in the Shaker $\mathrm{K}(+)$ channel. J Gen Physiol 115:123-138

Todt H, Dudley SC Jr, Kyle JW, French RJ, Fozzard HA (1999) Ultraslow inactivation in mu1 $\mathrm{Na}^{+}$channels is produced by a structural rearrangement of the outer vestibule. Biophys J 76:1335-1345

Tombola F, Pathak MM, Isacoff EY (2005a) How far will you go to sense voltage? Neuron 48:719-725

Tombola F, Pathak MM, Isacoff EY (2005b) Voltage-sensing arginines in a potassium channel permeate and occlude cation-selective pores. Neuron 45:379-388

Townsend C, Horn R (1997) Effect of alkali metal cations on slow inactivation of cardiac $\mathrm{Na}^{+}$channels. J Gen Physiol 110:23-33

Tu YH, Cooper AJ, Teng B, Chang RB, Artiga DJ, Turner HN, Mulhall EM, Ye W, Smith AD, Liman ER (2018) An evolutionarily conserved gene family encodes proton-selective ion channels. Science 359:1047-1050

Uysal S, Vasquez V, Tereshko V, Esaki K, Fellouse FA, Sidhu SS, Koide S, Perozo E, Kossiakoff A (2009) Crystal structure of full-length KcsA in its closed conformation. Proc Natl Acad Sci USA 106:6644-6649

Wainger BJ, DeGennaro M, Santoro B, Siegelbaum SA, Tibbs GR (2001) Molecular mechanism of cAMP modulation of HCN pacemaker channels. Nature 411:805-810

Wang SY, Wang GK (1997) A mutation in segment I-S6 alters slow inactivation of sodium channels. Biophys J 72:1633-1640

Wei R, Wang X, Zhang Y, Mukherjee S, Zhang L, Chen Q Huang X, Jing S, Liu C, Li S, Wang G, Xu Y, Zhu S, Williams AJ, Sun F, Yin C-C (2016) Structural insights into $\mathrm{Ca}(2+)$-activated longrange allosteric channel gating of RyR1. Cell Res 26:977-994

Whicher JR, MacKinnon R (2016) Structure of the voltage-gated $\mathrm{K}(+)$ channel Eag1 reveals an alternative voltage sensing mechanism. Science 353:664-669

Xu H, Ren D (2015) Lysosomal physiology. Annu Rev Physiol 77:57-80 
Yan Z, Zhou Q, Wang L, Wu J, Zhao Y, Huang G, Peng W, Shen H, Lei J, Yan N (2017) Structure of the Nav1.4-beta1 complex from electric eel. Cell 170:470-482

Yang N, Horn R (1995) Evidence for voltage-dependent S4 movement in sodium channels. Neuron 15:213-218

Yang HT, Hu MH, Guo JL, Ou XM, Cai TX, Liu ZF (2016) Pore architecture of TRIC channels and insights into their gating mechanism. Nature 538:537

Yellen G (2002) The voltage-gated potassium channels and their relatives. Nature 419:35-42

Yu FH, Yarov-Yarovoy V, Gutman GA, Catterall WA (2005) Overview of molecular relationships in the voltage-gated ion channel superfamily. Pharmacol Rev 57:387-395

Zarrabi T, Cervenka R, Sandtner W, Lukacs P, Koenig X, Hilber K, Mille M, Lipkind GM, Fozzard HA, Todt H (2010) A molecular switch between the outer and the inner vestibules of the voltage-gated $\mathrm{Na}^{+}$channel. J Biol Chem 285:39458-39470

Zhang X, Ren W, DeCaen P, Yan C, Tao X, Tang L, Wang J, Hasegawa K, Kumasaka T, He J, Wang J, Clapham DE, Yan N (2012) Crystal structure of an orthologue of the NaChBac voltagegated sodium channel. Nature 486:130-134
Zhang XC, Zhao Y, Heng J, Jiang D (2015) Energy coupling mechanisms of MFS transporters. Protein Sci 24:1560-1579

Zhang XC, Liu Z, Li J (2016) From membrane tension to channel gating: a principal energy transfer mechanism for mechanosensitive channels. Protein Sci 25:1954-1964

Zhang XC, Liu M, Han L (2017a) Energy coupling mechanisms of AcrB-like RND transporters. Biophys Rep 3:73-84

Zhang XC, Liu M, Lu G, Heng J (2017b) Thermodynamic secrets of multidrug resistance: a new take on transport mechanisms of secondary active antiporters. Protein Sci 27:595-613

Zhao Y, Scheuer T, Catterall WA (2004) Reversed voltagedependent gating of a bacterial sodium channel with proline substitutions in the S6 transmembrane segment. Proc Natl Acad Sci USA 101:17873-17878

Zheng J, Sigworth FJ (1998) Intermediate conductances during deactivation of heteromultimeric Shaker potassium channels. J Gen Physiol 112:457-474

Zhou Y, Morais-Cabral JH, Kaufman A, MacKinnon R (2001) Chemistry of ion coordination and hydration revealed by a $\mathrm{K}^{+}$ channel-Fab complex at $2.0 \AA \AA$ resolution. Nature 414:43-48 